\title{
Analytical Analysis for Optimizing Mass Ratio of Nonlinear Tuned Mass Dampers
}

\section{Tianjiao Zhang ( $063550181 @ q q . c o m$ )}

Dalian University of Technology

\section{Luyu Li}

Dalian University of Technology

\section{Research Article}

Keywords: tuned mass dampers, civil engineering, nonlinear coefficient, optimal mass ratio

Posted Date: May 13th, 2021

DOI: https://doi.org/10.21203/rs.3.rs-449889/v1

License: (c) (i) This work is licensed under a Creative Commons Attribution 4.0 International License. Read Full License

Version of Record: A version of this preprint was published at Nonlinear Dynamics on September 17th, 2021. See the published version at https://doi.org/10.1007/s11071-021-06845-x. 


\title{
Analytical Analysis for Optimizing Mass Ratio of Nonlinear Tuned Mass Dampers
}

\author{
Tianjiao Zhang', Luyu $\mathbf{L i}^{2}$ \\ ${ }^{1}$ School of construction engineering, Dalian University of Technology, Dalian, China. \\ ${ }^{2}$ School of construction engineering, Dalian University of Technology, Dalian, China \\ Corresponding author: \\ Tianjiao Zhang, School of construction engineering, Dalian University of Technology, Dalian, \\ Liaoning, 116024 China. \\ Email: 693550181@qq.com
}

\begin{abstract}
To reduce the adverse vibrations of buildings, tuned mass dampers (TMDs), which are the most representative passive control devices, have been widely used and studied in aerospace, machinery, civil engineering, and other fields for many years. Most scholars used to treat the TMD as a linear damper, but they show some nonlinear characteristics owing to the use of limit devices and large displacements. It is necessary to consider the nonlinear coefficient of the TMD when designing its parameters. In this study, the mass ratio of the TMD was optimized with considering the nonlinear coefficient of the TMD. The complex variable average method and multiscale method were used for analysis. A mass ratio interval was found on the " $\varepsilon-N_{2}$ " curve in which modulation response can occur, and then an analytical method for obtaining the optimal mass ratio of TMD was derived based on this phenomenon. The numerical results showed that taking the midpoint of this mass ratio interval as the optimal mass ratio can yield a better damping effect and robustness than using the traditional linear design method.
\end{abstract}

\section{Keywords}

tuned mass dampers, civil engineering, nonlinear coefficient, optimal mass ratio

\section{Introduction}

Unpredictable natural disasters and human-induced attacks pose a great threat to the 
safety of buildings; therefore, research on vibration reduction of structures is important in the field of civil engineering. Vibration control is mainly divided into active, semi-active, and passive control. The main difference between them is whether they require an external energy input. Among the control devices, passive control, with the simplest structure and the lowest cost, has been widely studied for many years [1-3], and the tuned mass damper (TMD) is the most representative. Its mechanical model can be simplified as an auxiliary mass and a controlled primary structure, which are connected by a linear spring and damper. Energy is transferred between the TMD and primary structure through resonance. TMD originated in 1911, and Frahmn invented a type of undamped TMD called a dynamic vibration absorber (DVA) in the same year. However, the control bandwidth was too narrow owing to the lack of damping, even worse than the uncontrolled structure on the other frequency bands except tuning [4]. Based on the above defects, Den Harton introduced damping into the DVA, which greatly improved the damping effect [5], and the concept of TMD was also established. He optimized the frequency ratio and damping ratio of the TMD and provided their specific optimization formula [6]. Tsai and Lin proposed an optimal parameter design method for TMDs based on Den Harton's formula [7]. Setareh et al. obtained the optimal damping of a TMD by using the integrated modal method [8].

However, TMD has certain limitations. For example, the control effect of the TMD depends on whether it is precisely tuned (tuning refers to the frequency of the TMD being adjusted to be consistent with the first mode of vibration of the primary structure). However, the frequency component of the excitation is very complex. The TMD will lose its efficiency or even make the vibration more intense when the system deviates from the 1:1 internal resonance. Therefore, scholars have begun to investigate this limitation of TMD and attempted to develop a more general and efficient TMD. For example, in 1988, Clark first extended a single-degree-of-freedom (DOF) TMD into a multi-degree-of-freedom one and proposed the concept of multiple tuned mass damper (MTMD) [9]. Alamnzan proposed a bidirectional homogeneous TMD (BH-TMD) [10]. HuJiuzhan et al. used a distributed TMD to 
reduce the vibrations of a multimode cable-stayed bridge. The configuration optimization and parameter optimization of the TMD were produced by the $\mathrm{H} 2$ norm based on modal coordinates and the gradient optimization method based on $\mathrm{H} 2$ properties, respectively [11].

Later, some scholars suggested that it would produce nonlinearity owing to the use of a limitation device or large displacement when TMD works [12, 13]. This implies that TMD is not really a purely linear system. Li and Cui analyzed the spontaneous nonlinearity in TMD and proposed that if this nonlinearity was ignored, it would cause adverse effects on vibration reduction. They simplified the system to one with a single degree of freedom in which the force of the primary structure acting on the TMD was regarded as an equivalent load, and they optimized the parameters of the TMD designed by the linear method [14]. Li and Zhang used the complex variable average method and multi-scale method to derive the optimal frequency formula of the TMD under the premise of considering the nonlinear coefficient of the TMD. The numerical results confirmed the superiority of the above formula [15].

The model studied in this paper is a 2-DOF system composed of a linear main structure under harmonic excitation and a TMD mass, which is connected by springs and dampers in parallel. This study focuses on the optimization of TMD's mass ratio. The paper is structured as follows. Section 1 introduces the research background of TMDs and the main content of this paper. Section 2 introduces the mechanical model of the system and its dimensionless form. In Section 3, the complex variable average method and multiscale method are used to remove the fast-varying part of the variable, the exact equation is transformed into the complex variable equation, and the slow-varying equation of the system is derived. Section 4 introduces the process and principle of the mass ratio optimization method, and a range of TMD mass ratios in which the modulation response can occur is obtained. In Section 5, by taking the mass ratio at the starting point and at the midpoint of the above interval as the mass ratio of TMD, respectively, and by comparing the control effects of these two cases, we concluded that the midpoint of the interval would provide stronger robustness as the optimal mass ratio of TMD. Finally, Section 6 summarizes the main content of this 
paper.

\section{Motion equations of the system}

The simplified model of the system considering the nonlinear coefficient of the TMD is shown in Figure 1. It should be noted that this nonlinear coefficient is not a design parameter, but an inevitable attribute of TMD due to the use of a limitation device or large displacement in the vibration process. In this section, we first establish a mechanical model for the system.

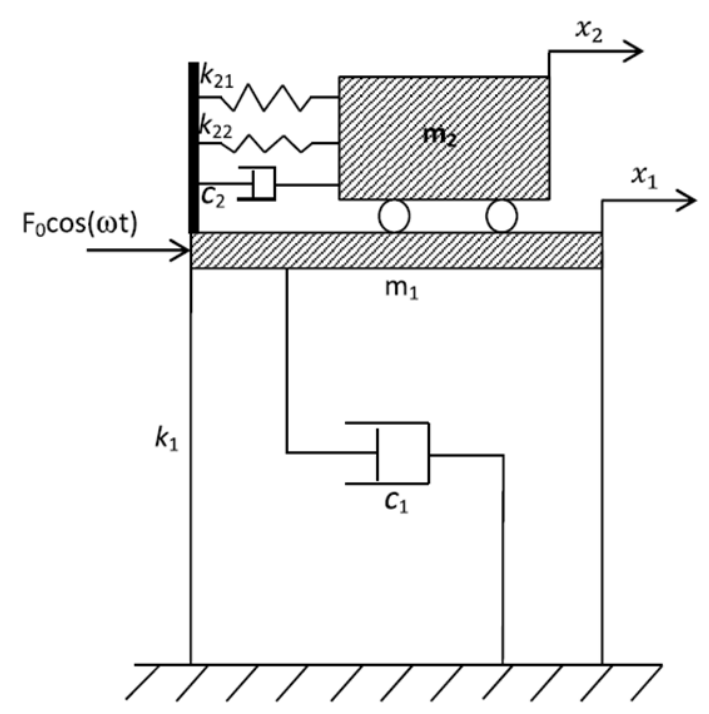

Fig. 1 Simplified model of the 2-DOF system

The equations of motion are expressed as follows:

$$
\begin{gathered}
m_{1}+c_{1} \cdot+k_{1} x_{1}+m_{2}=F \cos \omega t \\
m_{2}+c_{2}(\&-\&)+k_{21}\left(x_{2}-x_{1}\right)+k_{22}\left(x_{2}-x_{1}\right)^{3}=0
\end{gathered}
$$

To facilitate calculations, Equations (1) and (2) can be made dimensionless.

$$
\frac{m_{2}}{m_{1}}=\varepsilon, \frac{c_{1}}{m_{1}}=\varepsilon \lambda_{1}, \frac{k_{1}}{m_{1}}=\omega_{1}^{2}, \frac{F}{m_{1}}=\varepsilon f, \frac{k_{22}}{k_{21}}=\alpha, \frac{c_{2}}{m_{2}}=\lambda_{2}, \frac{k_{21}}{m_{2}}=\omega_{2}^{2}
$$

By substituting the dimensionless parameters into Equations (1) and (2), the dimensionless forms of the equation of motion are obtained as follows:

$$
\begin{gathered}
+\varepsilon \lambda_{1} \cdot d+\omega_{1}^{2} x_{1}+\varepsilon=\varepsilon f \cos \omega t \\
+\lambda_{2}(\underset{\&}{\&})+\omega_{2}^{2}\left(x_{2}-x_{1}\right)+\alpha \omega_{2}^{2}\left(x_{2}-x_{1}\right)^{3}=0
\end{gathered}
$$

The absolute displacements $x_{1}$ and $x_{2}$ of the primary structure and the TMD are 
transformed into relative displacements $u$ and $v$, respectively.

$$
u=x_{1}+\varepsilon x_{2}, v=x_{1}-x_{2}
$$

Substituting Equation (6) into Equations (4) and (5) allows us to establish the motion equations with relative coordinates.

$$
\begin{aligned}
& \frac{\varepsilon}{1+\varepsilon} \lambda_{1}\left(\imath \&+\varepsilon+\frac{\omega_{1}^{2}}{\varepsilon+1}(u+\varepsilon v)=\varepsilon f \cos \omega t\right. \\
& (\varepsilon+1) \lambda_{2} \&+(\varepsilon+1) \omega_{2}^{2} v+(\varepsilon+1) \alpha \omega_{2}^{2} v^{3}=0
\end{aligned}
$$

To smoothly carry out the next steps, the Taylor formula is used to change the equations into a form that only contains one second derivative in each equation. Equations (7) and (8) are regarded as polynomials of $\varepsilon$. Expanding Equations (7) and (8) near $\varepsilon=0$ yields the following:

$$
\mathrm{f}(\varepsilon)=\mathrm{f}(0)+\varepsilon \mathrm{f}^{\prime}(0)
$$

From Equation (9), we can rewrite Equations (7) and (8) as follows:

$$
\begin{gathered}
\omega_{1}^{2} u+\varepsilon\left(\lambda_{1} l \&-\omega_{1}^{2} u+\omega_{1}^{2} v-f \cos \omega t\right)=0 \\
\omega_{1}^{2} v+\lambda_{2} \&+\omega_{1}^{2}(u-v)+\omega_{2}^{2}\left(v+\alpha v^{3}\right)+\varepsilon\left(\lambda_{1} \iota \&+\lambda_{2}\right) \&-\omega_{1}^{2} u \\
\left.+\omega_{1}^{2} v+\omega_{2}^{2} v+\alpha \omega_{2}^{2} v^{3}-f \cos \omega t\right)=0
\end{gathered}
$$

Thus far, the equations of motion expressed in relative coordinates have been obtained. In later calculations, Equations (10) and (11) will be referred to as being the exact equations of the system.

\section{Analytical analysis}

In this section, we will use the complex variable average method and the multiscale method to analyze Equations (10) and (11) to eliminate the fast-varying and higher-order terms because they have minimal significance in the current study. Only the necessary slow-varying terms that we can deduce directly are retained.

Because this study only focuses on the response near 1:1:1 internal resonance, the natural frequency of the primary structure is defined as $\omega_{1}=1$, and the tuning parameter is defined as $\sigma$ to facilitate calculations. Then, the excitation frequency can be expressed as follows: 


$$
\omega=1+\varepsilon \sigma
$$

The real variables $u$ and $v$ are replaced by the complex variables $\varphi_{1}$ and $\varphi_{2}$, respectively, and $\varphi_{1}$ and $\varphi_{2}$ are expressed as

$$
\varphi_{1} e^{i \omega t}=\imath \&+i \omega u, \varphi_{2} e^{i \omega t}=1 \&+i \omega v
$$

After substituting Equation (13) into Equations (10) and (11), performing the average process, and omitting higher-order terms, the following equations are obtained:

$$
\begin{gathered}
\phi \&+\frac{\varepsilon}{2}\left(\lambda_{1} \varphi_{1}+i \varphi_{1}-i \varphi_{2}-f e^{i \varepsilon \sigma t}\right)=0 \\
\frac{\varepsilon}{2}\left(2 \phi \frac{\alpha}{2}+\lambda_{2} \varphi_{2}+i \varphi_{2}-i \varphi_{1}-i \omega_{2}^{2} \varphi_{2}-\frac{3}{4} i \alpha \omega_{2}^{2}\left|\varphi_{2}\right|^{2} \varphi_{2}=0\right)
\end{gathered}
$$

There is still a fast-varying term in Equation (14), that is, the exponential term $e^{i \varepsilon \sigma t}$. To eliminate it, we can set $\psi_{1}$ and $\psi_{2}$ as follows:

$$
\begin{gathered}
\psi \&+i \varepsilon \sigma \psi_{1}+\frac{\varepsilon}{2}\left(\lambda_{1} \psi_{1}+i \psi_{1}-i \psi_{2}-f\right)=0 \\
\frac{\varepsilon}{2}\left(2 \psi \&_{2}+2 i \varepsilon \sigma \psi_{2}+\lambda_{2} \psi_{2}+i \psi_{2}-i \psi_{1}-i \omega_{2}^{2} \psi_{2}-\frac{3}{4} \alpha i \omega_{2}^{2}\left|\psi_{2}\right|^{2} \psi_{2}\right)=0
\end{gathered}
$$

The derivation is expressed as follows: $D=\frac{d}{d t}$. Using the multiscale method, we can divide the time variable $t$ into two new independent time variables:

$$
t=t_{0}+\varepsilon t_{1}
$$

The derivatives can now be written as $D_{0}=\frac{\partial}{\partial t_{0}}, D_{1}=\frac{\partial}{\partial t_{1}}$, and $D=D_{0}+\varepsilon D_{1}$. The new time variables can be substituted into Equations (16) and (17).

$$
\begin{gathered}
D_{0} \psi_{1}+\varepsilon D_{1} \psi_{1}+i \varepsilon \sigma \psi_{1}+\frac{\varepsilon}{2}\left(\lambda_{1} \psi_{1}+i \psi_{1}-i \psi_{2}-f\right)=0 \\
\frac{\varepsilon}{2}\left[2\left(D_{0} \psi_{2}+\varepsilon D_{1} \psi_{2}\right)+2 i \varepsilon \sigma \psi_{2}+\lambda_{2} \psi_{2}+i \psi_{2}-i \psi_{1}-i \omega_{2}^{2} \psi_{2}-\frac{3}{4} i \alpha \omega_{2}^{2}\left|\psi_{2}\right|^{2} \psi_{2}\right]=0
\end{gathered}
$$

Consider Equations (19) and (20) as polynomial equations about $\varepsilon$, and then the 
coefficient of each term can be expressed as follows:

$$
\begin{gathered}
\varepsilon^{0}: D_{0} \psi_{1}=0 \\
\varepsilon^{1}: D_{1} \psi_{1}+i \sigma \psi_{1}+\frac{1}{2}\left(\lambda_{1} \psi_{1}+i \psi_{1}-i \psi_{2}-f\right)=0 \\
\frac{1}{2}\left(2 D_{0} \psi_{2}+\lambda_{2} \psi_{2}+i \psi_{2}-i \psi_{1}-i \omega_{2}^{2} \psi_{2}-\frac{3}{4} i \alpha \omega_{2}^{2}\left|\psi_{2}\right|^{2} \psi_{2}\right)=0
\end{gathered}
$$

To verify that the analytical method selected in this study is sufficiently accurate, Figures 2-7 show the time history figures of the primary structure and TMD, which were obtained by simulating Equations (10), (11), (16), (17), (22), and (23) with the parameters $\alpha=0.003, \sigma=0, F=0.5, \lambda_{1}=5, \lambda_{2}=0.3, k_{21}=0.0194$, and the mass ratios of TMD are $\varepsilon=0.02,0.03,0.04$. As shown in the figures, the analytical method used in this study is effective because the curves from the three equations are in agreement. Although there are some minor deviations in the phase between complex variable equations and exact equations when the modulation response occurred, those can be ignored as the focus of this study is amplitude.

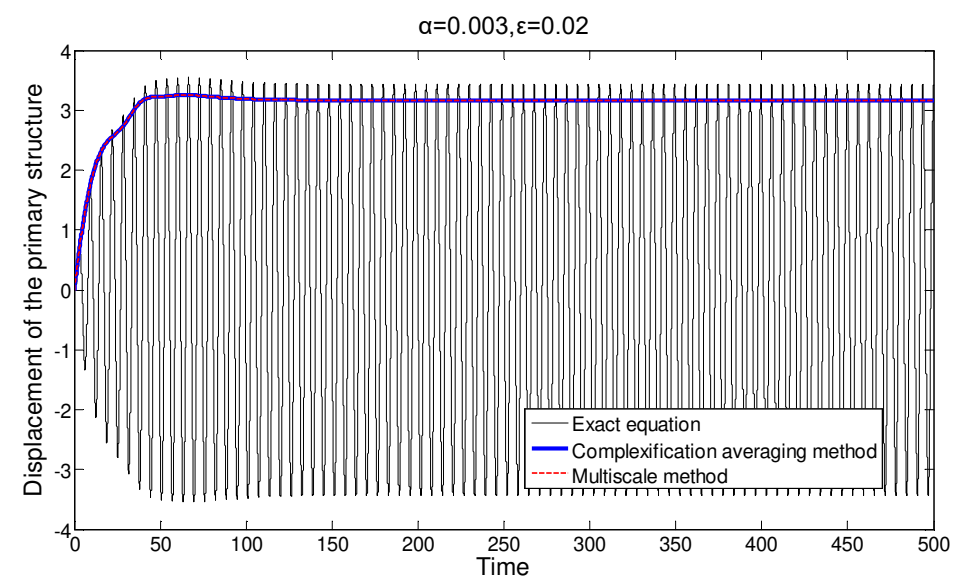

Fig. 2 Time-history curves of the primary structure for the case of $\varepsilon=0.02$ 


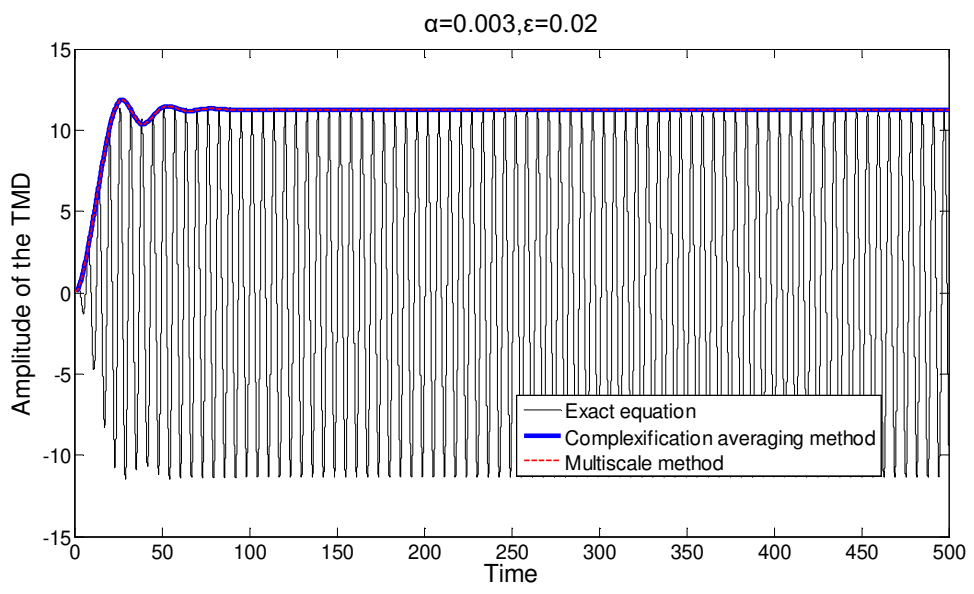

Fig. 3 Time-history curves of the TMD for the case of $\varepsilon=0.02$

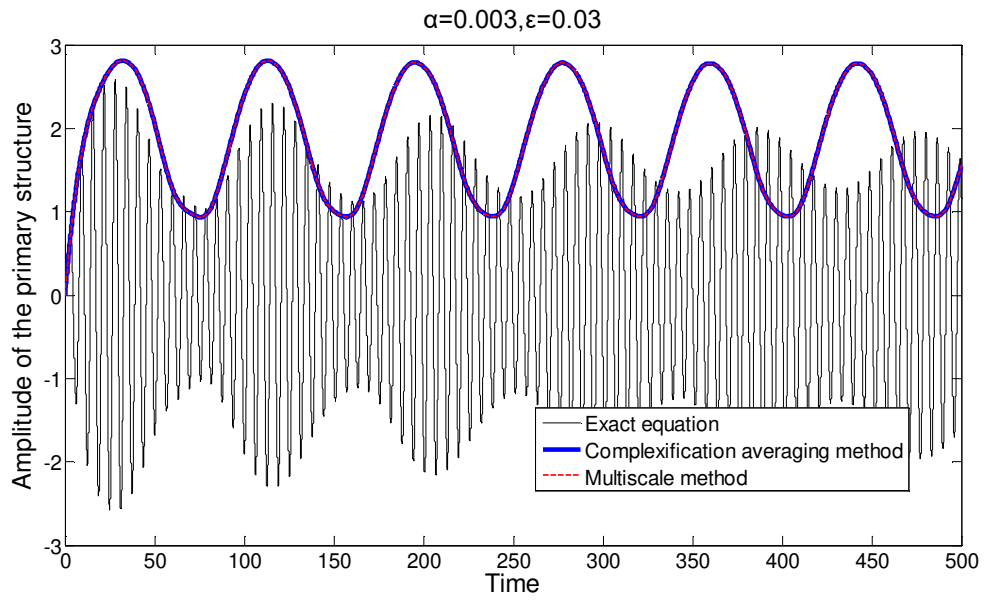

Fig. 4 Time-history curves of the primary structure for the case of $\varepsilon=0.03$

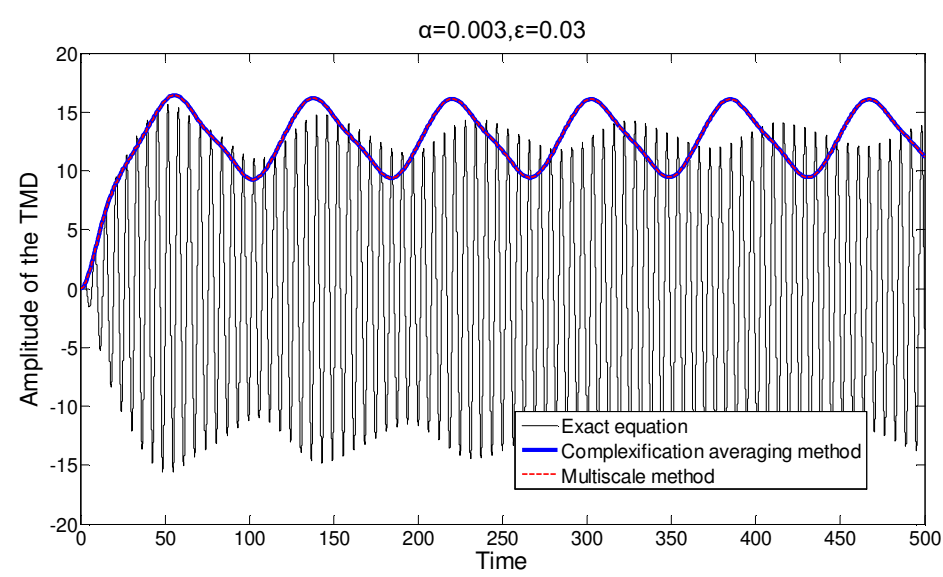

Fig. 5 Time-history curves of the TMD for the case of $\varepsilon=0.03$ 


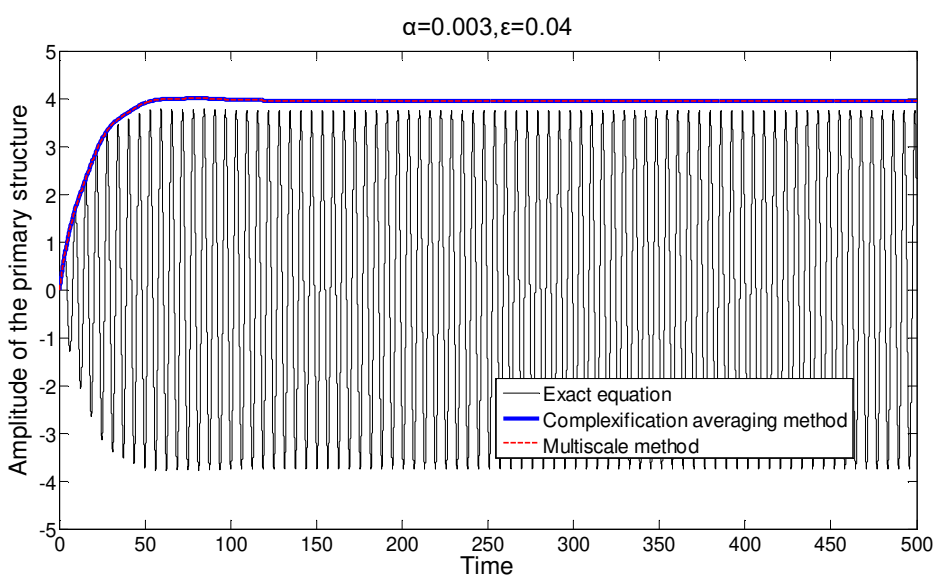

Fig. 6 Time-history curves of the primary structure for the case of $\varepsilon=0.04$

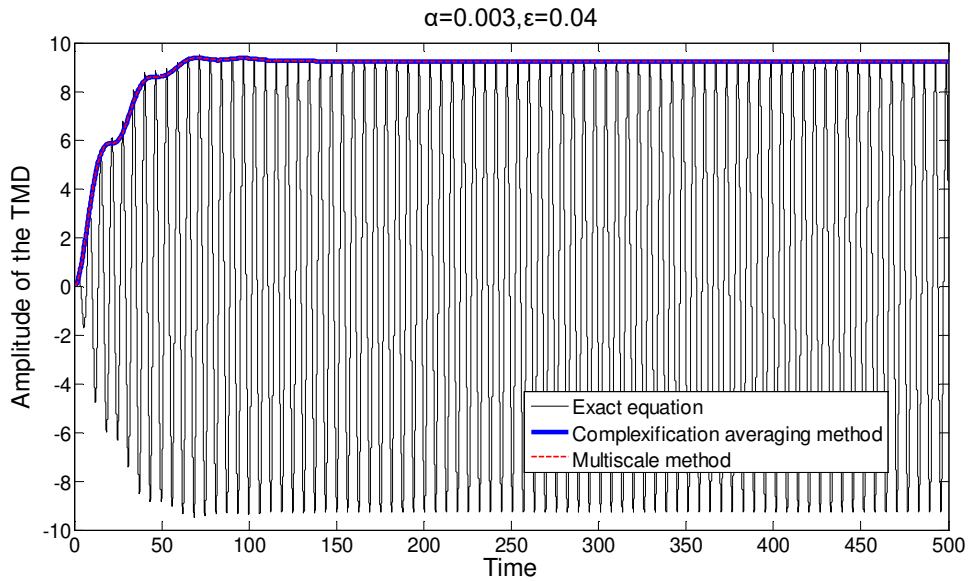

Fig. 7 Time-history curves of the TMD for case $\varepsilon=0.04$

Because we only studied the steady-state part of the response and the steady-state response was assumed to be stable on a fast-varying scale $\tau_{0}$, the derivative of Equation (23) can be assumed to be 0 . Thus, the slow invariant manifold (SIM) of this system can be obtained as follows:

$$
\psi_{1}=\psi_{2}\left(1-i \lambda_{2}-i \omega_{2}^{2}-\frac{3}{4} \alpha \omega_{2}^{2}\left|\psi_{2}\right|^{2}\right)
$$

The complex variables in Equation (24) can be transformed into an exponential form. Among them, $N_{1}$ and $N_{2}$ represent the slow-varying amplitude of the primary structure and the TMD, respectively, whereas $\delta_{1}$ and $\delta_{2}$ represent the phases of the primary structure and the TMD, respectively.

$$
\psi_{1}=N_{1} e^{i \delta_{1}}, \psi_{2}=N_{2} e^{i \delta_{2}}
$$


Let the square of the amplitude be

$$
Z_{1}=N_{1}^{2} ; Z_{2}=N_{2}^{2}
$$

By substituting Equations (25) and (26) into Equation (24) and separating the equation into real and imaginary parts, the following equation is obtained:

$$
Z_{1}=Z_{2}\left[\left(1-\omega_{2}^{2}-\frac{3}{4} \alpha \omega_{2}^{2} Z_{2}\right)^{2}+\lambda_{2}^{2}\right]
$$

To find the extreme points of the SIM, we derive $Z_{2}$ from Equation (21) and take its derivative as 0 .

$$
\lambda_{2}^{2}+\left(1-\omega_{2}^{2}-\frac{3 \alpha \omega_{2}^{2}}{4} Z_{2}\right)\left(1-\omega_{2}^{2}-\frac{9 \alpha \omega_{2}^{2}}{4} Z_{2}\right)=0
$$

Two local extreme points are obtained, which can also be called saddle-node bifurcation ( $\mathrm{SN}$ bifurcation) points or jump points.

$$
\begin{aligned}
& Z_{21}=\frac{4}{9 \alpha \omega_{2}^{2}}\left[2\left(1-\omega_{2}^{2}\right)-\sqrt{\left(1-\omega_{2}^{2}\right)^{2}-3 \lambda_{2}^{2}}\right] \\
& Z_{22}=\frac{4}{9 \alpha \omega_{2}^{2}}\left[2\left(1-\omega_{2}^{2}\right)+\sqrt{\left(1-\omega_{2}^{2}\right)^{2}-3 \lambda_{2}^{2}}\right]
\end{aligned}
$$

In practice, jumping occurs when the points depend on the initial condition. The parameters $\quad \alpha=0.003, k_{21}=0.0194, c_{1}=0.1, c_{2}=0.0028, F=0.5, \sigma=0 \quad$ were $\quad$ kept constant, and the mass ratios were taken as $\varepsilon=0.002,0.003,0.004$, respectively. The SIMs obtained from Equation (27) are shown in Figures 8, 9, and 10.

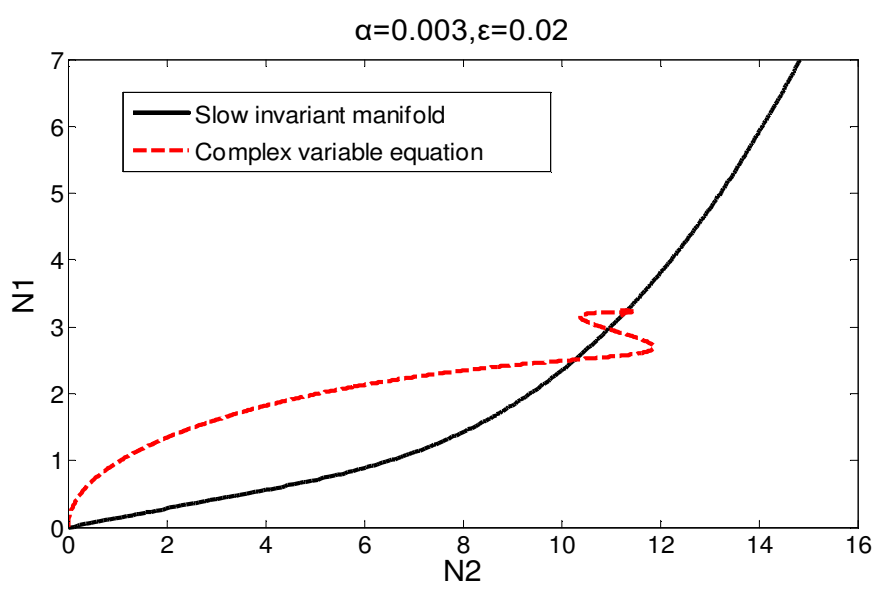

Fig. 8 Slow invariant manifold for the case of $\varepsilon=0.02$ 


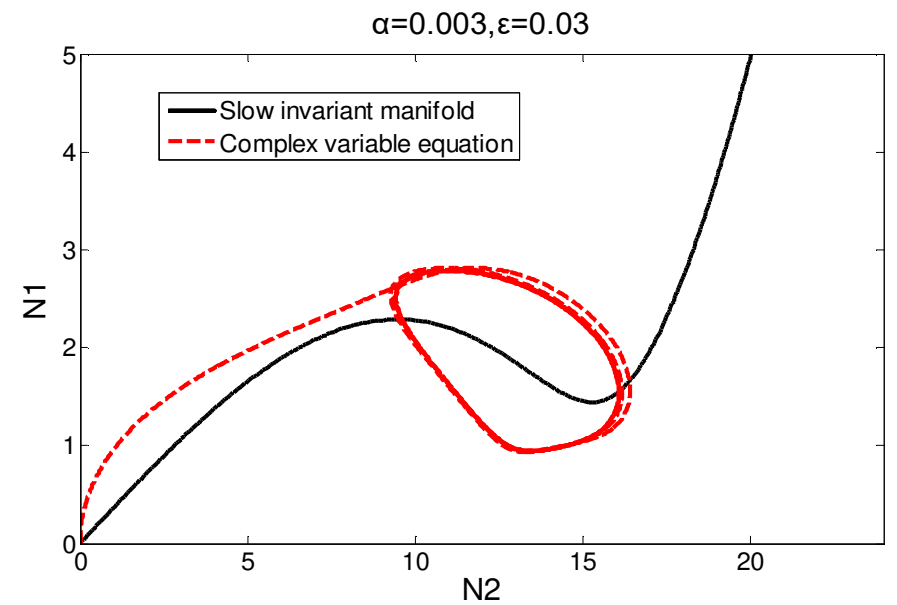

Fig. 9 Slow invariant manifold for the case of $\varepsilon=0.03$

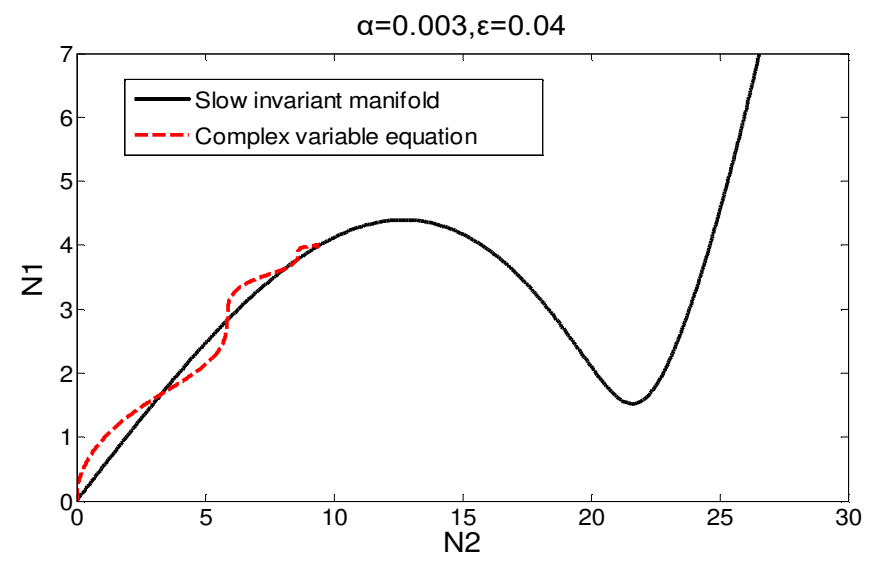

Fig. 10 Slow invariant manifold for the case of $\varepsilon=0.04$

By observing and comparing the three SIMs, we can see that there is a jump phenomenon in the weakly nonlinear TMD. However, it is clear that the occurrence of jumping is conditional, and the response mode changes with the change in $\varepsilon$. As shown in Figures 8-10, the jump phenomenon can only be observed in Figure 9, and the response mode is the modulation response. There was no jump phenomenon in $\varepsilon=0.02$ or $\varepsilon=0.04$. Figures $1-6$ show the corresponding time-history curves of the three SIMs, from which the response modes can be observed more clearly. This shows that the response mode does not change linearly with an increase in the mass ratio, and the modulation response only occurs in a certain range of mass ratios. To further analyze the change law of the response mode with the mass ratio, slow-varying equations should be deduced. Substituting Equation (24) into Equation (22) yields the following: 


$$
\begin{aligned}
& \left(1-\omega_{2}^{2}-\lambda_{2} i-\frac{3}{2} \alpha \omega_{2}^{2}\left|\psi_{2}\right|^{2}\right) \frac{\partial \psi_{2}}{\partial \tau_{1}}-\frac{3}{4} \alpha \omega_{2}^{2} \psi_{2}^{2} \frac{\partial \psi_{2}^{*}}{\partial \tau_{1}} \\
& +\frac{\psi_{2}}{2}\left[\lambda_{1}+2 i \sigma-\left(\lambda_{1}+2 i \sigma+i\right)\left(\omega_{2}^{2}+\lambda_{2} i+\frac{3}{4} \alpha \omega_{2}^{2}\left|\psi_{2}\right|^{2}\right)\right]=\frac{f}{2}
\end{aligned}
$$

Equation (31) can be rewritten more conveniently as follows:

$$
\frac{\partial \psi_{2}}{\partial \tau_{1}}=\frac{A^{*} G-B G^{*}}{|A|^{2}-|B|^{2}}
$$

$A, B$, and $G$ are defined as follows:

$$
\begin{gathered}
A=1-\lambda_{2} i-\omega_{2}^{2}-\frac{3}{2} \alpha \omega_{2}^{2}\left|\psi_{2}\right|^{2} \\
B=-\frac{3}{4} \alpha \omega_{2}^{2} \psi_{2}^{2} \\
G=\frac{f}{2}-\frac{\psi_{2}}{2}\left[\lambda_{1}+2 i \sigma-\left(\lambda_{1}+2 i \sigma+i\right)\left(\omega_{2}^{2}+\lambda_{2} i+\frac{3}{4} \alpha \omega_{2}^{2}\left|\psi_{2}\right|^{2}\right)\right]
\end{gathered}
$$

Writing $\psi_{2}$ in exponential form, substituting it into Equation (32), and separating the real part from the imaginary part yields the following:

$$
\begin{gathered}
\frac{\partial N_{2}}{\partial \tau_{1}}=\frac{1}{2 M}\left[\left(1-\omega_{2}^{2}-\frac{3}{4} \alpha \omega_{2}^{2} N_{2}^{2}\right) C+\lambda_{2} D\right] \\
\frac{\partial \delta_{2}}{\partial \tau_{1}}=\frac{1}{2 M N_{2}}\left[\lambda_{2} C-\left(1-\omega_{2}^{2}-\frac{9}{4} \alpha \omega_{2}^{2} N_{2}^{2}\right) D\right]
\end{gathered}
$$

$C, D$, and $M$ are defined as follows:

$$
\begin{gathered}
C=f \cos \delta_{2}-N_{2}\left[\lambda_{1}\left(1-\omega_{2}^{2}-\frac{3}{4} \alpha \omega_{2}^{2} N_{2}^{2}\right)+\lambda_{2}(2 \sigma+1)\right] \\
D=f \sin \delta_{2}+N_{2}\left[2 \sigma-\lambda_{1} \lambda_{2}-(2 \sigma+1)\left(\omega_{2}^{2}+\frac{3}{4} \alpha \omega_{2}^{2} N_{2}^{2}\right)\right] \\
M=\lambda_{2}^{2}+\left(1-\omega_{2}^{2}-\frac{3}{4} \alpha \omega_{2}^{2} N_{2}^{2}\right)\left(1-\omega_{2}^{2}-\frac{9}{4} \alpha \omega_{2}^{2} N_{2}^{2}\right)
\end{gathered}
$$

Equations (40) and (41) can be rewritten as follows:

$$
\frac{\partial N_{2}}{\partial \tau_{1}}=\frac{f_{1}\left(\delta_{2}, N_{2}\right)}{2 M}
$$




$$
\frac{\partial \delta_{2}}{\partial \tau_{1}}=\frac{f_{2}\left(\delta_{2}, N_{2}\right)}{2 M N_{2}}
$$

When the steady state response is stable, the following is true:

$$
f_{1}=f_{2}=0
$$

Equation (43) can be rewritten as

$$
\left[\begin{array}{ll}
\alpha_{11} & \alpha_{12} \\
\alpha_{21} & \alpha_{22}
\end{array}\right]\left[\begin{array}{l}
\cos \delta_{2} \\
\sin \delta_{2}
\end{array}\right]=\left[\begin{array}{l}
\beta_{1} \\
\beta_{2}
\end{array}\right]
$$

The variables $P$ and $Q$ can be defined as follows

$$
\begin{aligned}
& P=N_{2}\left[\lambda_{1}\left(1-\omega_{2}^{2}-\frac{3}{4} \alpha \omega_{2}^{2} N_{2}^{2}\right)+\lambda_{2}(2 \sigma+1)\right] \\
& Q=N_{2}\left[2 \sigma-\lambda_{1} \lambda_{2}-(2 \sigma+1)\left(\omega_{2}^{2}+\frac{3}{4} \alpha \omega_{2}^{2} N_{2}^{2}\right)\right]
\end{aligned}
$$

Then, the coefficient matrix in Equation (44) can be written as

$$
\begin{gathered}
\alpha_{11}=\left(1-\omega_{2}^{2}-\frac{3}{4} \alpha \omega_{2}^{2} N_{2}^{2}\right) f \\
\alpha_{12}=\alpha_{21}=\lambda_{2} f \\
\alpha_{22}=-\left(1-\omega_{2}^{2}-\frac{9}{4} \alpha \omega_{2}^{2} N_{2}^{2}\right) f \\
\beta_{1}=\left(1-\omega_{2}^{2}-\frac{3}{4} \alpha \omega_{2}^{2} N_{2}^{2}\right) P-\lambda_{2} Q \\
\beta_{2}=\lambda_{2} P+\left(1-\omega_{2}^{2}-\frac{9}{4} \alpha \omega_{2}^{2} N_{2}^{2}\right) Q
\end{gathered}
$$

The solution of Equation (44) can be written as follows:

$$
\begin{gathered}
\cos \delta_{2}=\frac{\alpha_{22} \beta_{1}-\alpha_{12} \beta_{2}}{\alpha_{11} \alpha_{22}-\alpha_{12} \alpha_{21}} \\
\sin \delta_{2}=\frac{\alpha_{11} \beta_{2}-\alpha_{21} \beta_{1}}{\alpha_{11} \alpha_{22}-\alpha_{12} \alpha_{21}}
\end{gathered}
$$

Substituting Equations (48) and (49) into the trigonometric identity, the following frequency response equation can be obtained: 


$$
\left[\lambda_{1}\left(1-\omega_{2}^{2}-\frac{3}{4} \alpha \omega_{2}^{2} N_{2}^{2}\right)+\lambda_{2}(2 \sigma+1)\right]^{2}+\left[2 \sigma-\lambda_{1} \lambda_{2}-(2 \sigma+1)\left(\omega_{2}^{2}+\frac{3}{4} \alpha \omega_{2}^{2} N_{2}^{2}\right)\right]^{2}=\frac{f^{2}}{N_{2}^{2}}
$$

To analyze the frequency response of Equation (50), the tuning parameter $\varepsilon$ is first approximated as being 0. Then, Equation (50) can be rewritten as follows:

$$
\left\{\left[\lambda_{1}\left(1-\omega_{2}^{2}-\frac{3}{4} \alpha \omega_{2}^{2} N_{2}^{2}\right)+\lambda_{2}\right]^{2}+\left(\omega_{2}^{2}+\frac{3}{4} \alpha \omega_{2}^{2} N_{2}^{2}+\lambda_{1} \lambda_{2}\right)^{2}\right\} N_{2}^{2}-f^{2}=0
$$

In the later analysis of the slow-varying equations, because the denominator $M$ is equal to the derivative of the SIM (i.e., $M=\frac{\partial N_{1}}{\partial N_{2}}$ ), it will result in a singularity at the jumping point, causing the system to not be simulated normally. To address this problem, rescaling the time by the term $M$ without affecting the analysis results [16-20] yields the following equations:

$$
\begin{aligned}
& N_{2}^{\prime}=f_{1}\left(\delta_{2}, N_{2}\right) \\
& \delta_{2}^{\prime}=f_{2}\left(\delta_{2}, N_{2}\right)
\end{aligned}
$$

where ' is the derivative with respect to the rescaled time.

\section{Optimization analysis of mass ratio}

In reference [12], the optimal frequency function of the TMD was successfully derived and applied to the design of the TMD (please refer to [12] for the full derivation). However, it is necessary to adjust the natural frequency of the TMD by adjusting its mass ratio. This section will attempt to analyze the mass ratio of the TMD directly and observe the change in amplitude with variations in the mass ratio of TMD. First, we use exact Equations (10) and (11), complex variable Equations (16) and (17), and super slow-varying Equations (52) and (53) to obtain the relationship curve between the mass ratio $\varepsilon$ and the square of the amplitude $N_{1}^{2}$ and $N_{2}^{2}$, as shown in Figures 11-14. The system parameters can be taken as $k_{21}=0.0194, c_{1}=0.1, c_{2}=0.0028, F=0.5, \sigma=0$. Under the condition of keeping the above parameters unchanged, nonlinear coefficients are taken 
$\alpha=0.003,0.004,0.005,0.006$.

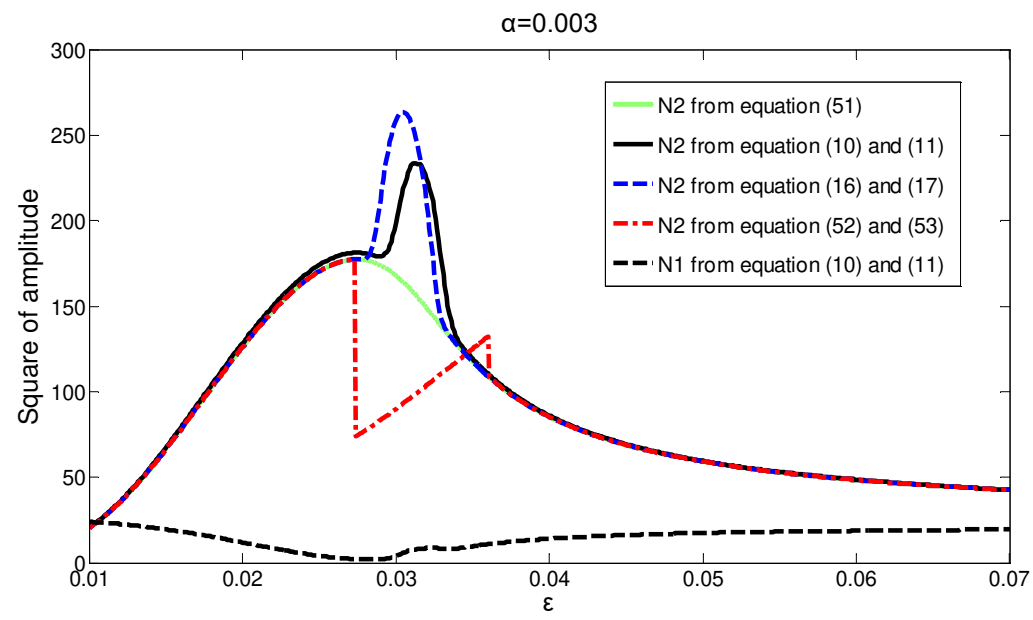

Fig. 11 Relationship between mass ratio and amplitudes for the case of $\alpha=0.003$

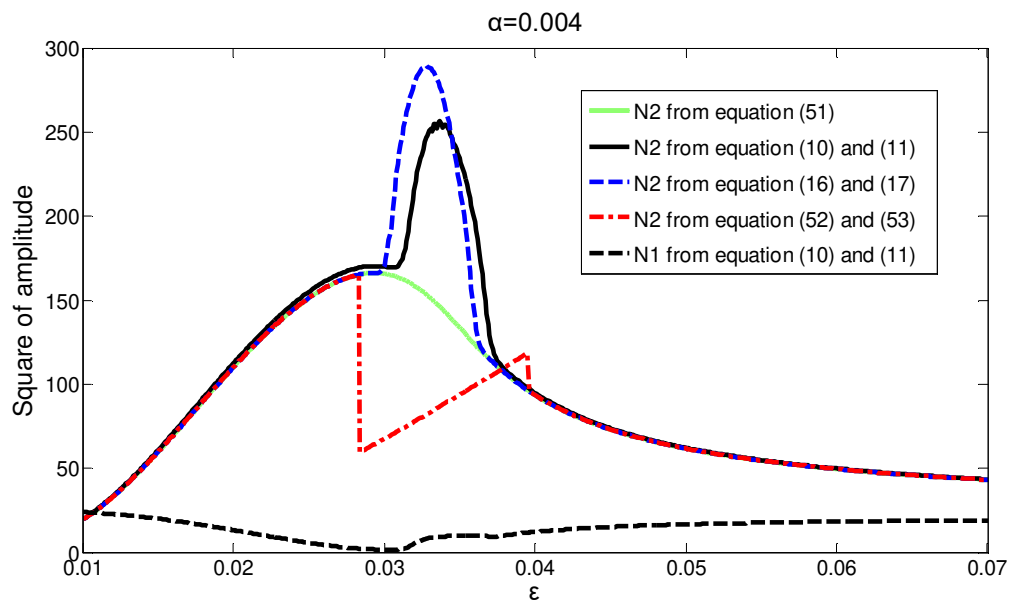

Fig. 12 Relationship between mass ratio and amplitudes for the case of $\alpha=0.004$

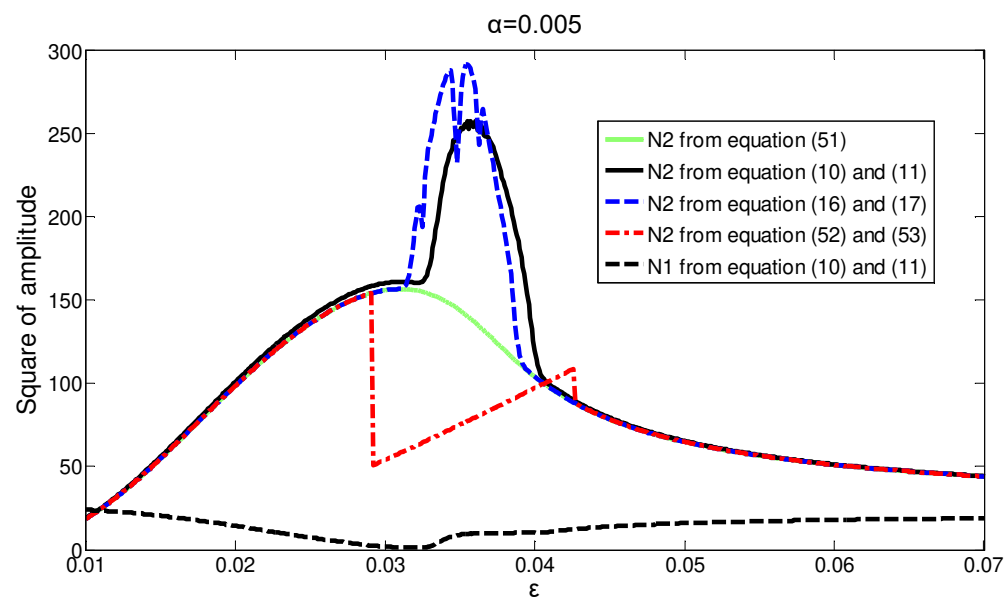

Fig. 13 Relationship between mass ratio and amplitudes for the case of $\alpha=0.005$ 


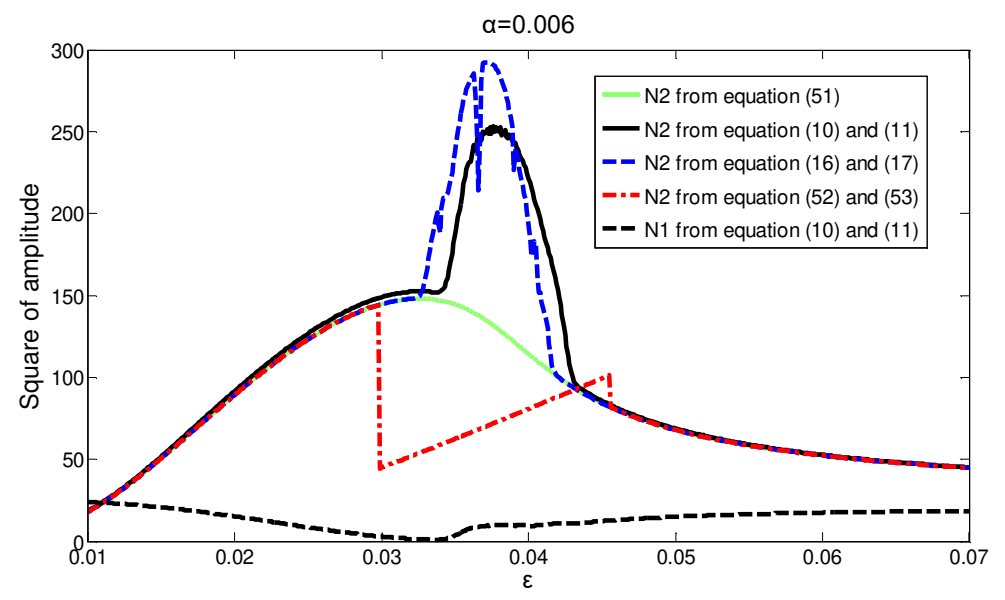

Fig. 14 Relationship between mass ratio and amplitudes for the case of $\alpha=0.006$

As shown in these figures, the " $\varepsilon-N_{2}^{2}$ " curve has a minimum point. The abscissa corresponding to this point is the TMD mass ratio, which minimizes the steady-state amplitude of the primary structure. It seems that this mass ratio corresponding to the minimum point should be regarded as the optimal mass ratio of the TMD. However, it should be noted that Figures 11-14 are only for the case of $\omega=1$. In fact, the excitation frequency fluctuates and cannot be constant. Therefore, the analysis in the frequency domain is necessary.

As shown in Figures 11-14, the abscissa of the maximum point of $N_{2}^{2}$ and the minimum point of $N_{1}$ almost coincide, which can also be understood from the perspective of energy conservation. Therefore, we can transform the goal of finding the minimum point of $N_{1}^{2}$ to finding the maximum point of $N_{2}^{2}$. We can observe from Figures 10-13 that the "curve" simulated by the exact, complex variable, and super slow-varying equations basically coincides with the curve drawn by the continuous Equation (51), except for small convex or concave ranges, and the abscissa of these parts almost coincide.

This is evidently not an accidental calculation error. Equation (51) is based on the stable steady-state response, but when the modulation response occurs, the steady-state response is unstable. Thus, the derivative is not equal to 0 , and the calculation result of Equation (51) is no longer accurate at this time. Therefore, it can 
be speculated that the abscissa regions corresponding to the convex or concave parts are in the TMD mass ratio range where the modulation response can occur. This conjecture will be verified by the numerical method.

Taking the parameters as being $k_{21}=0.0194, c_{1}=0.1, c_{2}=0.0028, F=0.5, \sigma=0, \alpha=0.003$, the time-history curves of displacement are obtained using Equations (10) and (11), complex variable Equations (16) and (17), and slow-varying Equations (52) and (53), respectively, as shown in Figures 15-17.

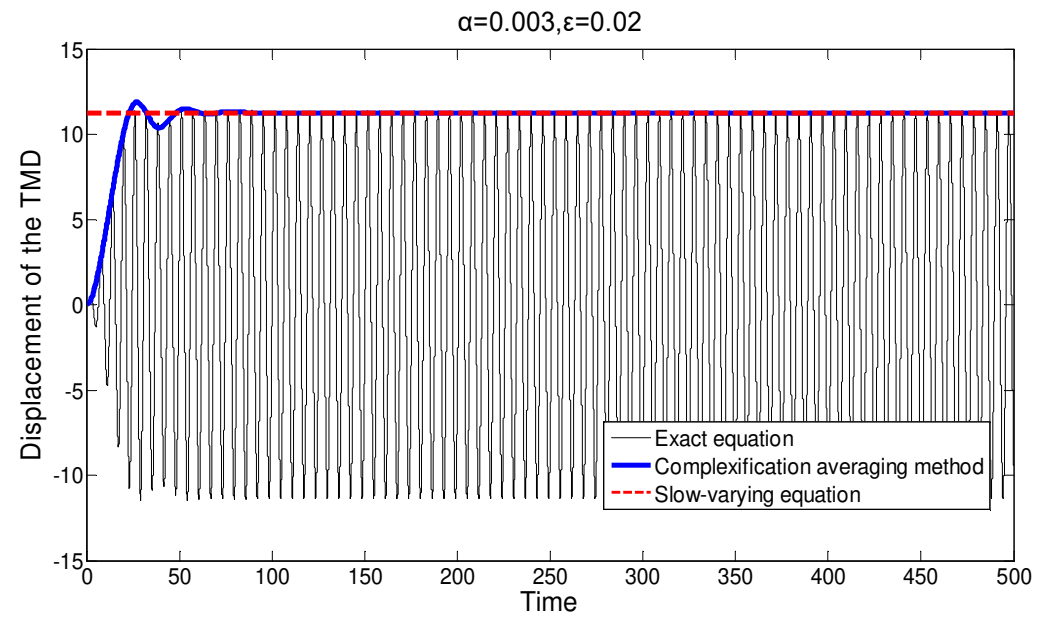

Fig. 15 Time-history curves of the primary structure for the case of $\varepsilon=0.02$

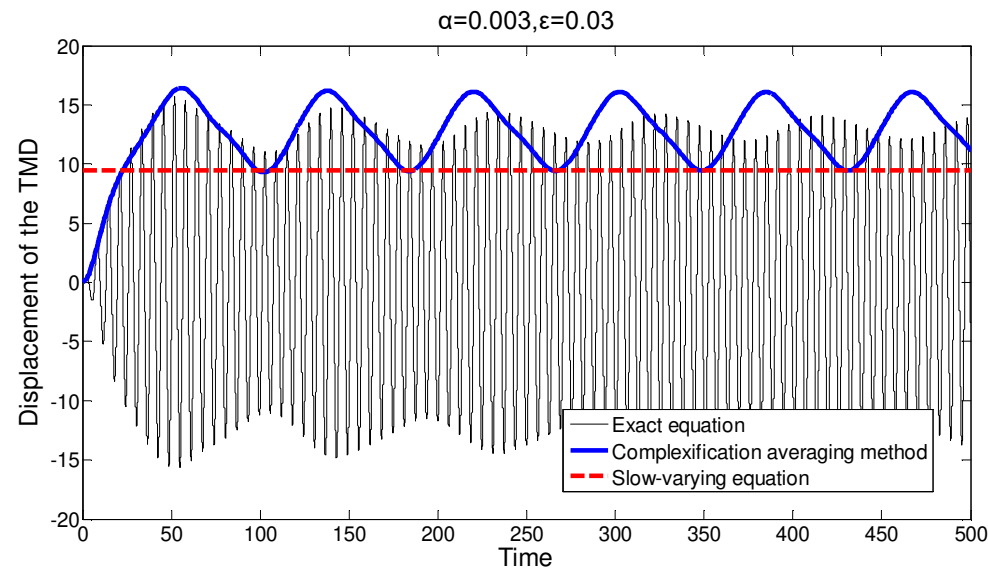

Fig. 16 Time-history curves of the primary structure for the case of $\varepsilon=0.03$ 


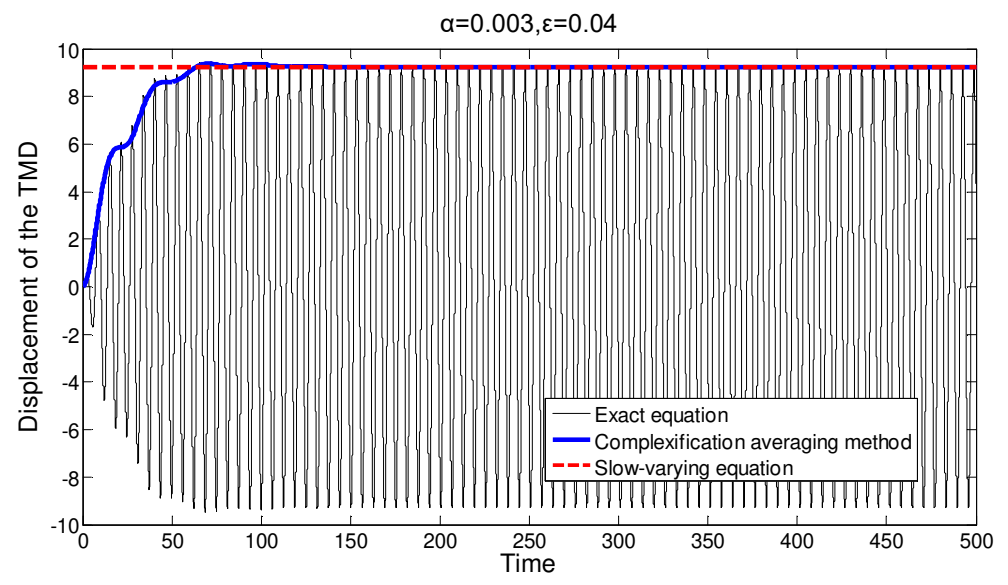

Fig. 17 Time-history curves of the primary structure for the case of $\varepsilon=0.04$

Figure 15 shows that, when $\varepsilon=0.02$, the steady-state response of the system is stable, and the time history curves obtained from the three equations are in good agreement. As shown in Figure 7, there is no jump point at this time, so there is no modulation response. When $\varepsilon=0.03$, the steady-state response of the system is a modulation response, which can also be observed from the corresponding SIM (Figure 8). Although there are some errors in their convex or concave parts between the three equations, they do not affect the analysis results and, thus, can be ignored. In the concave part, $N_{2}$ is only equal to the amplitude at the jump point of the SIM, rather than the real steady-state amplitude of TMD. This is because the modulation response is generated by the continuous jump, but the jump belongs to the instantaneous fast-varying behavior. Hence, the slow-varying equations that ignore the fast-varying variable are no longer accurate when the modulation response occurs. It is also the case that the slow-varying equations are based on the premise that the derivative of $N_{2}$ with respect to $\tau_{0}$ is 0 . However, $N_{2}$ cannot be fixed when the modulation response occurs, which implies that the derivative $\frac{\partial N_{2}}{\partial \tau_{0}}$ is no longer equal to 0 at this time. However, the slow-varying equations are still accurate before the jump occurs. Therefore, the SIM made by the slow-varying equations will stay at the jumping point $N_{21}$ (or $N_{22}$ ) after experiencing instantaneous growth. It can be observed from Figure 10 that when the mass ratio is 0.04 , there is no jump in the 
system, the steady-state response from Figure 17 is stable, and the three curves are in good agreement again. The above analysis confirmed the conjecture that the steady-state response mode in the inconsistent interval is the modulation response, and the steady-state response is stable in the other coinciding part.

To further verify the above inference, another larger nonlinear coefficient can be taken as 0.005 , and let $\varepsilon=0.025,0.035,0.045$. The same conclusion can be drawn from Figures 18-20. It has been proved that the inconsistent interval of the " $\varepsilon-N_{2}^{2}$ " curves is the interval where modulation response occurs, which implies that the starting points and ending points of this interval are the starting points and ending points of the jump, respectively. It can be inferred that the values of $N_{2}$ at these two points are equal to the amplitudes at the jump points on the SIMs. Then, we attempt to find the range of $\varepsilon$ where the modulation response occurs according to the above analysis. Equation (51) can be transformed into a form expressed by $\varepsilon$ using the following substitutions:

$$
\omega_{2}^{2}=\frac{k_{21}}{m_{2}}, \lambda_{1}=\frac{c_{1}}{m_{2}}, \lambda_{2}=\frac{c_{2}}{m_{2}}, f=\frac{F}{m_{2}}, \varepsilon=\frac{m_{2}}{m_{1}}=\frac{m_{2}}{1}=m_{2}
$$

Set the parameter $Y$ as follows:

$$
Y=k_{21}\left(1+\frac{3}{4} \alpha N_{2}^{2}\right)
$$

By substituting Equations (54) and (55) into Equation (51), it can be rewritten as

$$
\left\{\left[\varepsilon\left(c_{1}+c_{2}\right)-c_{1} Y\right]^{2}+\left(\varepsilon Y+c_{1} c_{2}\right)^{2}\right\} N_{2}^{2}=\varepsilon^{2} F^{2}
$$

The derivative of Equation (56) with respect to $\varepsilon^{2}$ is as follows (this step is to find the extremum of " $\varepsilon-N_{2}$ " curve):

$$
N_{2}^{2}\left[\varepsilon^{2}\left(c_{1}+c_{2}\right)^{2}+\varepsilon^{2} k_{21}^{2}\left(1+\frac{3}{4} \alpha y\right)^{2}-k_{21} c_{1}^{2}\left(1+\frac{3}{4} \alpha N_{2}^{2}\right)\right]=\varepsilon^{2} F^{2}
$$

The relationship between $\varepsilon$ and $N_{2}$ at the extreme point of the " $\varepsilon-N_{2}^{2}$ " curve can be obtained by simplifying Equation (57) 


$$
\varepsilon=\sqrt{\frac{c_{1}^{2} N_{2}^{2} Y}{N_{2}^{2}\left(c_{1}+c_{2}\right)^{2}+N_{2}^{2} Y^{2}-F^{2}}}
$$

The value of $N_{2}$ at the extreme point can be obtained by substituting Equation (58) into Equation (56). Then, the mass ratio of TMD at the extreme point can be obtained by substituting this $N_{2}$ into Equation (58).

By substituting the jump point amplitude from Equation (27) into Equation (58), two solutions of $\varepsilon$ are obtained (because the initial conditions in civil engineering are mostly zero or minimal, the jumping point considered in this study is $N_{21}$ ), which are the mass ratios of the starting point and the ending point of the inconsistent interval. Although there are some small errors due to the approximate calculation among the original, complex variable, and slow-varying equations, the inconsistent interval is not completely coincident with each other, but it can be ignored because it does not affect the optimization effect.

Table 1 Interval of $\varepsilon$ in which the modulation response can occur (obtained from Equations (56) and (58))

\begin{tabular}{llllll}
\hline Nonlinear coefficient & 0.003 & 0.004 & 0.005 & 0.006 & 0.007 \\
\hline Starting point & 0.0277 & 0.0295 & 0.0311 & 0.0326 & 0.0340 \\
Ending point & 0.0345 & 0.0377 & 0.0406 & 0.0432 & 0.0457 \\
\hline
\end{tabular}

Table 2 Interval of $\varepsilon$ in which the modulation response can occur (taken from the curves of " $\varepsilon-N_{2}^{2}$ ”)

\begin{tabular}{llllll}
\hline Nonlinear coefficient & 0.003 & 0.004 & 0.005 & 0.006 & 0.007 \\
\hline Starting point & 0.0288 & 0.0307 & 0.0323 & 0.3390 & 0.0350 \\
Ending point & 0.0343 & 0.0377 & 0.0406 & 0.0434 & 0.0458 \\
\hline
\end{tabular}

Table 1 shows the modulation response interval calculated by Equations (56)-(58), and Table 2 shows the modulation response interval displayed on the curve when the nonlinear coefficients between 0.003 and 0.007 . Comparing the data in the two tables, it can be observed that, although there is a small error, the modulation 
response interval calculated by Equations (56)-(58) is reliable.

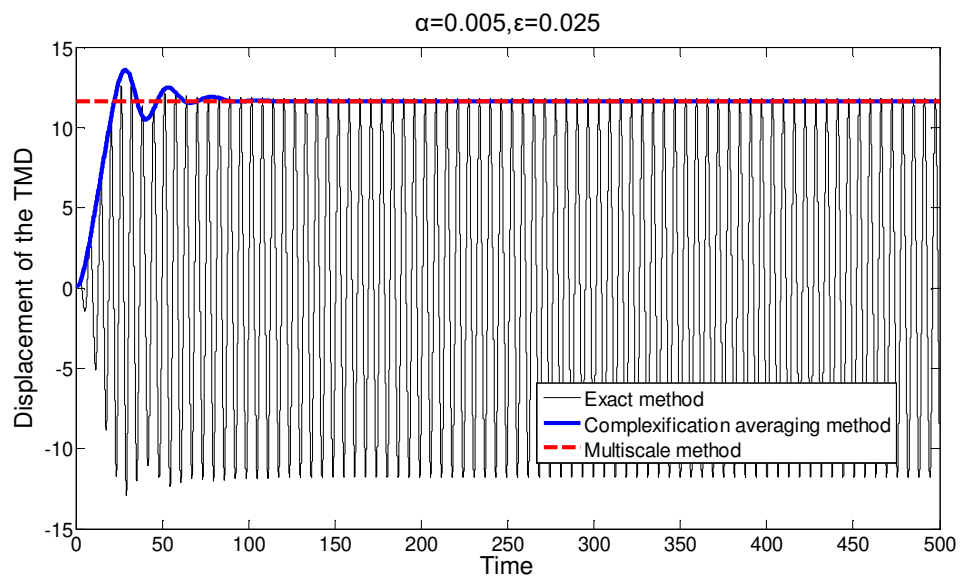

Fig. 18 Time-history curves of the primary structure for the case of $\alpha=0.005, \varepsilon=0.025$

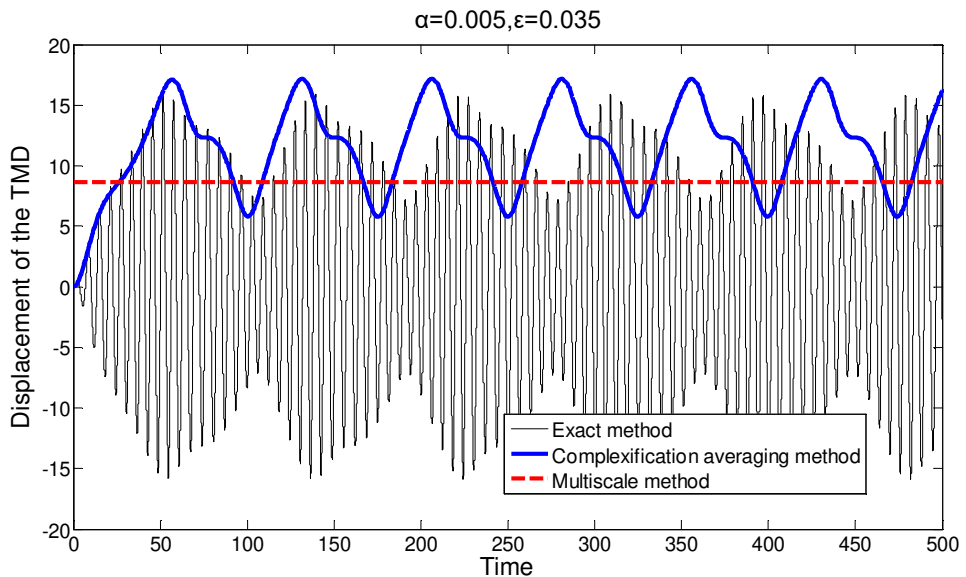

Fig. 19 Time-history curves of the primary structure for the case of $\alpha=0.005, \varepsilon=0.035$

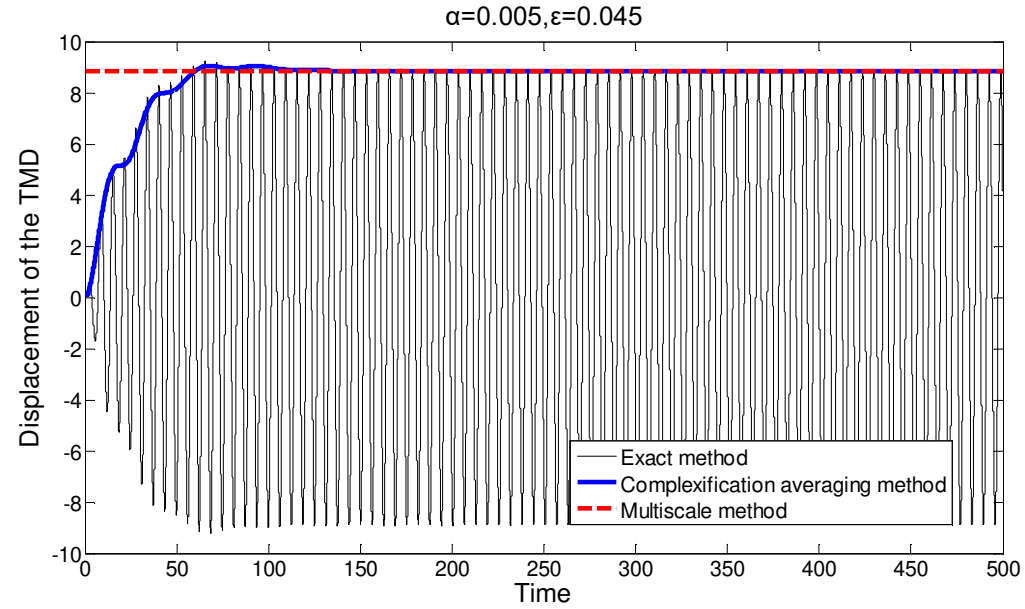

Fig. 20 Time-history curves of the primary structure for the case of 


$$
\alpha=0.005, \varepsilon=0.045
$$

Now, what needs to be considered is which point on the " $\varepsilon-N_{2}^{2}$ " curve should be regarded as the optimal mass ratio when the nonlinear coefficient is determined. Two schemes are considered: the first scheme takes the starting point of the modulation response, whereas the other one takes the midpoint of the modulation response.

The reason for taking the starting point is that it almost coincides with the minimum of $N_{1}$. However, as shown in Figures 11-14, the inconsistent intervals of the exact, complex variable, and slow-varying equations are not completely coincident with each other. In other words, there will be some errors, which lead to the poor robustness of the starting point. When the input condition is slightly changed, the response mode may significantly change. Based on this, considering the midpoint of the inconsistent interval provides higher robustness. On the other hand, the modulation response stimulates the occurrence of target energy transfer, and the TMD can be regarded as a nonlinear energy sink. The energy almost does not return to the primary structure after it is transferred to the TMD because of the instantaneous resonance caused by the nonlinear characteristics of the TMD. This mode can improve the energy-dissipation efficiency. In the next section, the optimization effects of the above two schemes are compared using a numerical method.

\section{Comparison of the control effect}

In this section, a numerical method is used to test the control effect of the above two optimal mass ratio schemes. The parameters in Figures 21-23 were $k_{21}=0.0194, c_{1}=0.1, c_{2}=0.0028, F=0.5$. From these, the frequency response curve of the primary structure when the nonlinear coefficient are $\alpha=0.003,0.005,0.007$, respectively. The control effect of the TMD when the mass ratio is at the starting point or the midpoint of the inconsistent interval on the " $\varepsilon-N_{2}^{2}$ " curve can be compared. The linear method used in this study was the fixed-point theory ${ }^{[4]}$. 


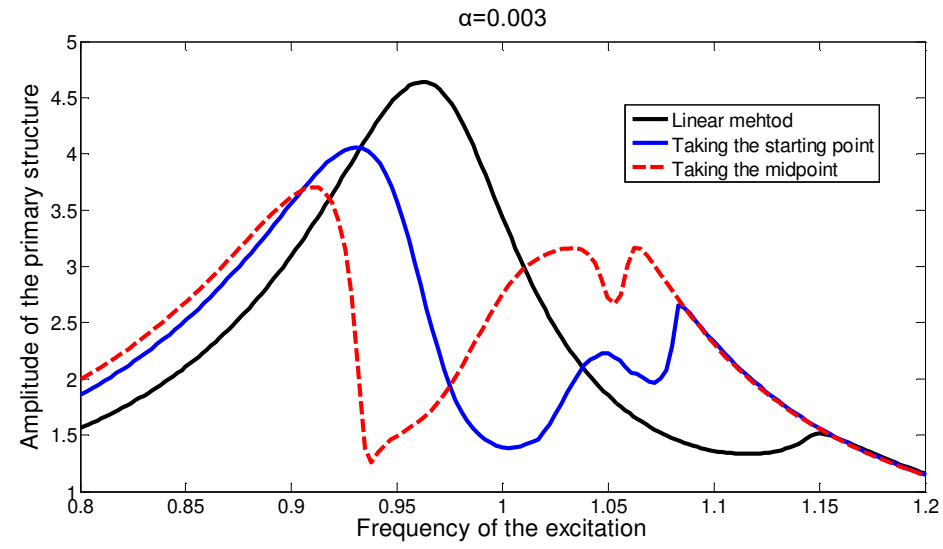

Fig. 21 Frequency response curve of primary structure for the case of $\alpha=0.003$

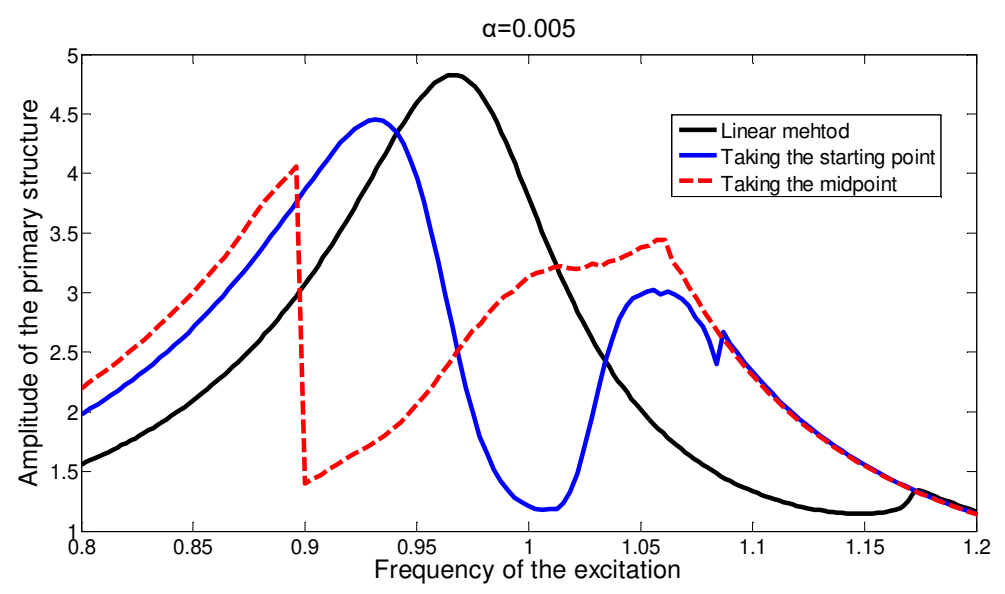

Fig. 22 Frequency response curve of primary structure

For the case of $\alpha=0.005$

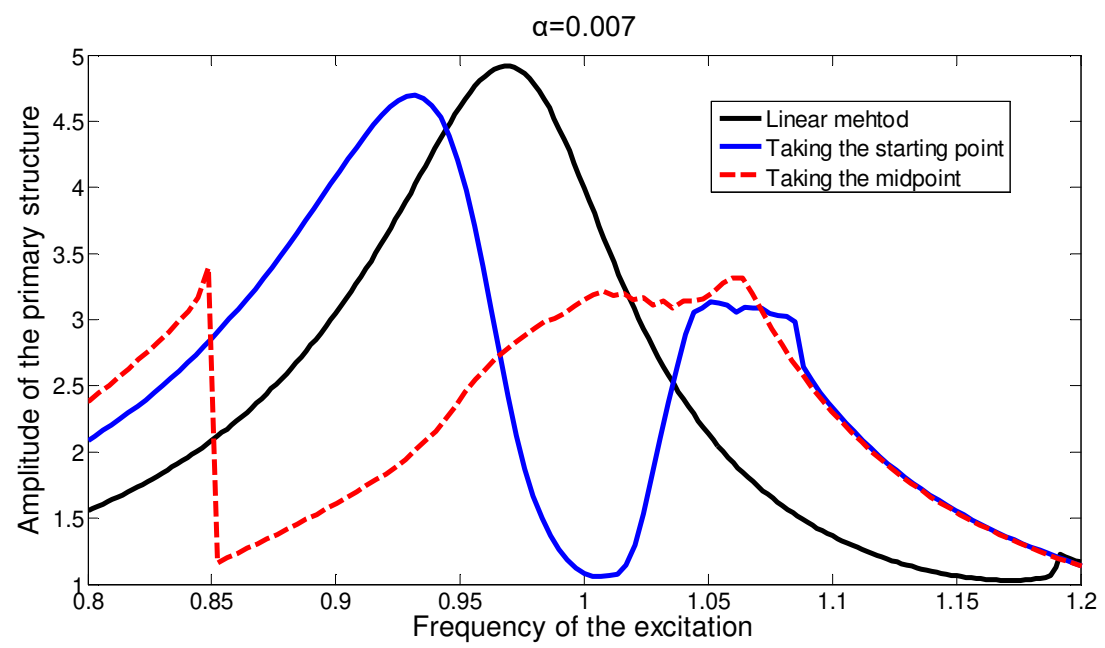

Fig. 23 Frequency response curve of primary structure for the case of $\alpha=0.007$ 
From the results in the frequency domain, it can be observed that the amplitudes are smaller when the starting points are $\omega=1$. However, when $\omega \neq 1$, it is better to take the midpoint because $N_{1}$ is more stable near $\omega=1$ when taking the midpoint and the amplitude fluctuation of the primary is relatively small. This is because the response mode is always modulated around the midpoint and the amplitude is more robust. However, the input condition is substantially complex in practice, and the frequency of excitation cannot always be equal to 1 , so the robustness in the frequency domain is crucial. According to the above analysis, the mass ratio at the midpoint of the modulation response interval is regarded as the optimal mass ratio of the TMD.

\section{Conclusion}

This paper presents an analysis of a 2-DOF system under harmonic excitation comprising a linear primary structure and a TMD using the complex variable average and multiscale methods. An analytical method for optimizing the mass ratio of the TMD was proposed on the premise of considering the nonlinear characteristics of the TMD. The effectiveness of the method was verified using a numerical method. This study can be divided into the following three stages:

1. The mechanical model of the system was established, and the slow-varying equations of the system were derived, which provided the possibility for the following optimization process. The correctness of the equation was verified by a numerical method. The analytical methods used in this study were proven to be sufficiently accurate.

2. When studying the " $\varepsilon-N_{2}^{2}$ " curve, it was found that the analytical and numerical solutions were partially inconsistent owing to the occurrence of the modulation response. Based on this, the interval of the TMD mass ratio that can produce the modulation response was deduced, and two schemes for selecting the optimal mass ratio of TMD were preliminarily proposed.

3. The numerical results showed that the above two schemes were much better than the linear method in terms of the control effect. Further, $\varepsilon$ at the midpoint of the 
interval at which the modulation response occurred with higher robustness was finally determined as the optimal quality ratio of the TMD through a comparison of the frequency domain.

\section{Acknowledgments}

This study is supported in part by the National Natural Science Foundation of China No.51678116.

\section{Data Availability Statement}

Some or all data, models, or code that support the findings of this study are available from the corresponding author upon reasonable request.

\section{Declaration of interests}

The authors declare that they have no known competing financial interests or personal relationships that could have appeared to influence the work reported in this paper.

\section{Reference}

1. Yang, Y.Z., Li, C.X.: Performance of tuned tandem mass dampers for structures under the ground acceleration. Structural Control \& Health Monitoring. 24, e1974 (2017).

2. Keshtegar, Behrooz, Etedali, Sadegh.: Nonlinear mathematical modeling and optimum design of tuned mass dampers using adaptive dynamic harmony search algorithm. Structural Control and Health Monitoring. 25(7), e2163 (2018)

3. Chung, L.L., Wu, L.Y., Yang, C., Lien, K.H., Lin, M.C., Huang, H.H.: Optimal design formulas for viscous tuned mass dampers in wind-excited structures. Structural Control \& Health Monitoring. 20(3), 320-336 (2013)

4. Hermann, F.: Device for Damping Vibrations of Bodies. U.S. Patent No. 989958 (1909)

5. Hartog, Den, J.P.: Mechanical Vibrations. McGraw-Hill Book Company, Inc, New York (1947)

6. Hartog, Den, J.P.: Mechanical Vibrations. McGraw-Hill Book Company, Inc, New York (1956)

7. Tsai, H.C., Lin, G.C.: Optimum tuned mass damper for minimizing steady-state response of support-excited and damped systems. Earthquake Eng. Struct. Dyn. 22, 957-973 (1993)

8. Setareh, M., Hanson, R.D.: Tuned Mass Dampers To Control Floor Vibration From Humans. Journal of Structural Engineering. 118(3), 741-762 (1992)

9. Clark, A.J.: Multiple Passive Tuned Mass Damper for Reducing Earth-quake 
Induced Building Motion. Pro 9th World Conf on Earth-quake Engrg. 283-290 (1988)

10. Almazan, J.L., Llera, J., Inaudi, J.A., Lopez-Garcia, D., Izquierdo, L.E.: A bidirectional and homogeneous tuned mass damper: a new device for passive control of vibrations. Engineering Structures. 29(7), 1548-1560 (2007)

11. Hu, J.Z.: Study on Placement and Parametric Optimization of Distributed TMDs for Seismic Control of Cable-stayed Bridge[分布式 TMD 对斜拉桥减震的配置 和参数优化研究]. Master thesis, Beijing Jiaotong University, Beijing (2014)

12. Teng, J., Lu, Z.X., Xiao, Y.Q., et al.: Vibration control of high-rising buildings with TMD considering contact nonlinear damping effect. Journal of Vibration and Shock. 28(3), 90-97 (2009)

13. Fallahpasand, S., Dardel, M., Pashaei, M.H., et al.: Investigation and optimization of nonlinear pendulum vibration absorber for horizontal vibration suppression of damped system. The Structural Design of Tall and Special Buildings. 24(14), 873-893 (2015)

14. Li, L.Y., Cui, P.: Novel design approach of a nonlinear tuned mass damper with duffing stiffness. Journal of Engineering Mechanics. 143(4), 04017004 (2017)

15. Li, L.Y., Zhang, T.J.: Analytical analysis for the design of nonlinear tuned mass damper. Journal of Vibration and Control. 26(10), 107754631988984 (2020)

16. Gendelman, O.V.: Bifurcations of Nonlinear Normal Modes of Linear Oscillator with Strongly Nonlinear Damped Attachment. Nonlinear Dynamics. 37(2), 115-128 (2004)

17. Gendelman, O.V., Starosvetsky, Y.: Quasi-Periodic Response Regimes of Linear Oscillator Coupled to Nonlinear Energy Sink Under Periodic Forcing. Journal of Applied Mechanics. 74(2), 325-331 (2007)

18. Gendelman, O.V., Starosvetsky, Y., Feldman, M.: Attractors of harmonically forced linear oscillator with attached nonlinear energy sink I: Description of response regimes. Nonlinear Dynamics. 51(1-2), 31-46 (2008)

19. Starosvetsky, Y., Gendelman, O.V.: Strongly modulated response in forced 2DOF oscillatory system with essential mass and potential asymmetry. PHYSICA D. 237(13), 1719-1733 (2008)

20. Starosvetsky, Y., Gendelman, O.V.: Response regimes of linear oscillator coupled to nonlinear energy sink with harmonic forcing and frequency detuning. Journal of Sound and Vibration. 315(3), 746-765 (2008) 
Figures

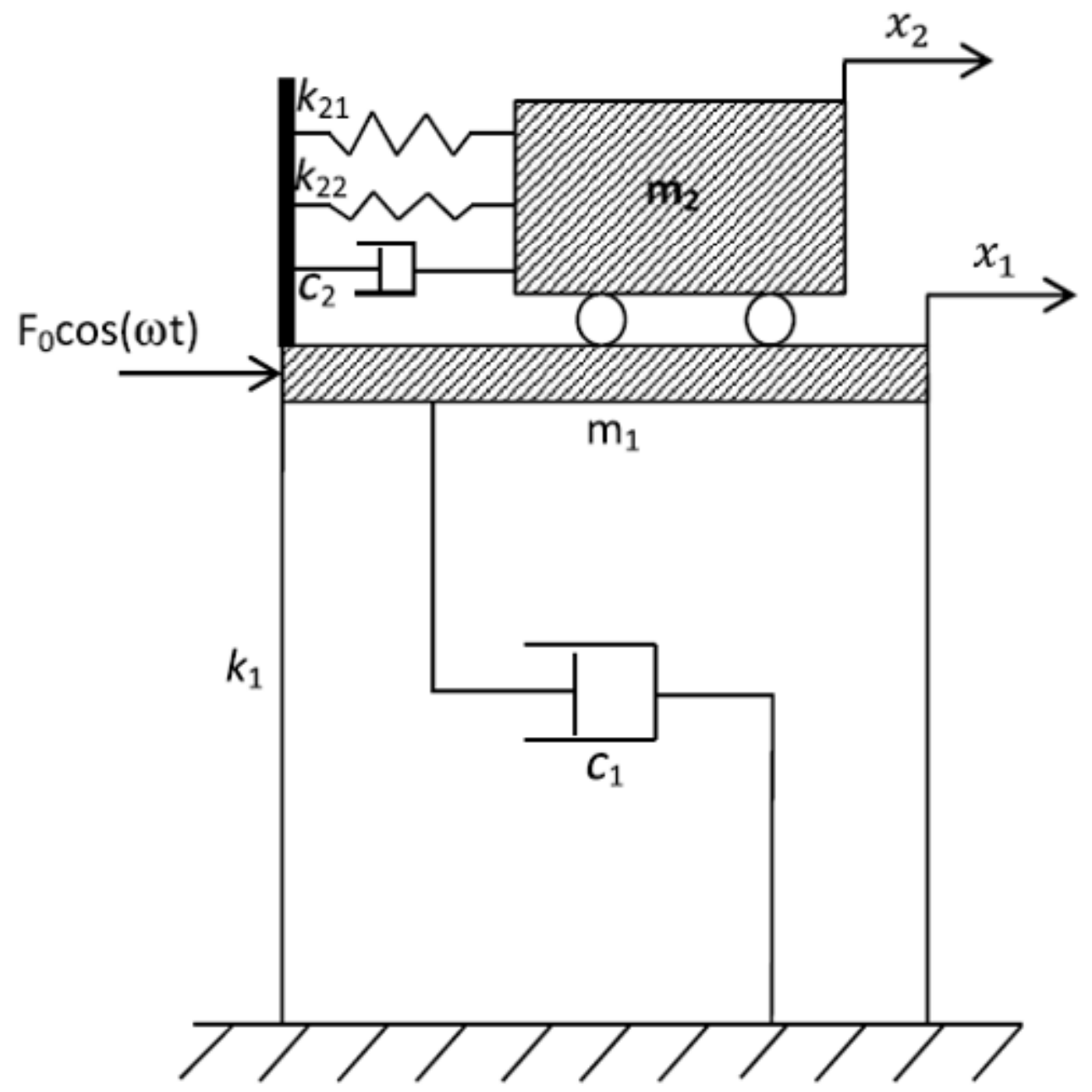

Figure 1

Simplified model of the 2-DOF system 


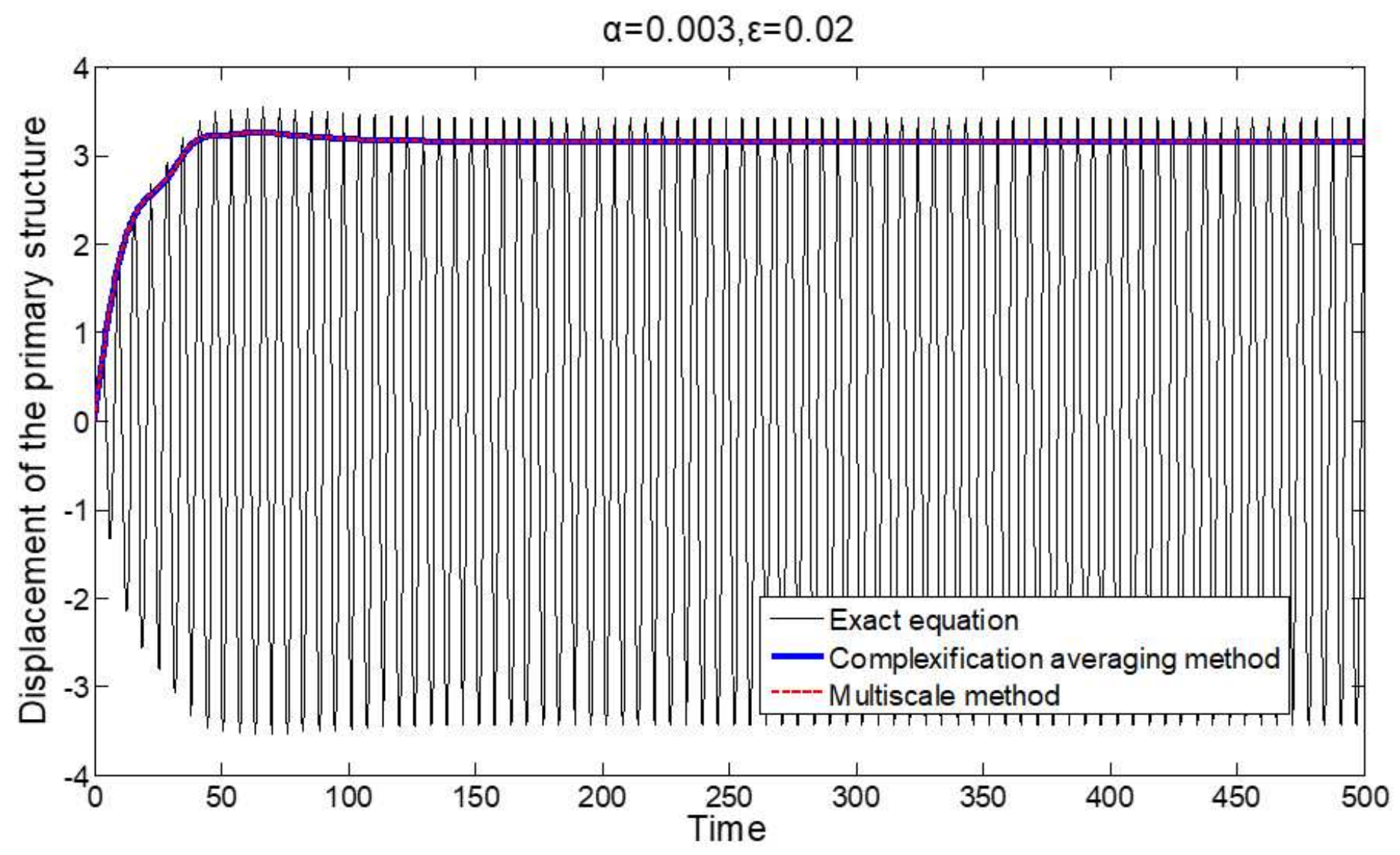

Figure 2

Time-history curves of the primary structure for the case of $\varepsilon=0.02$ 


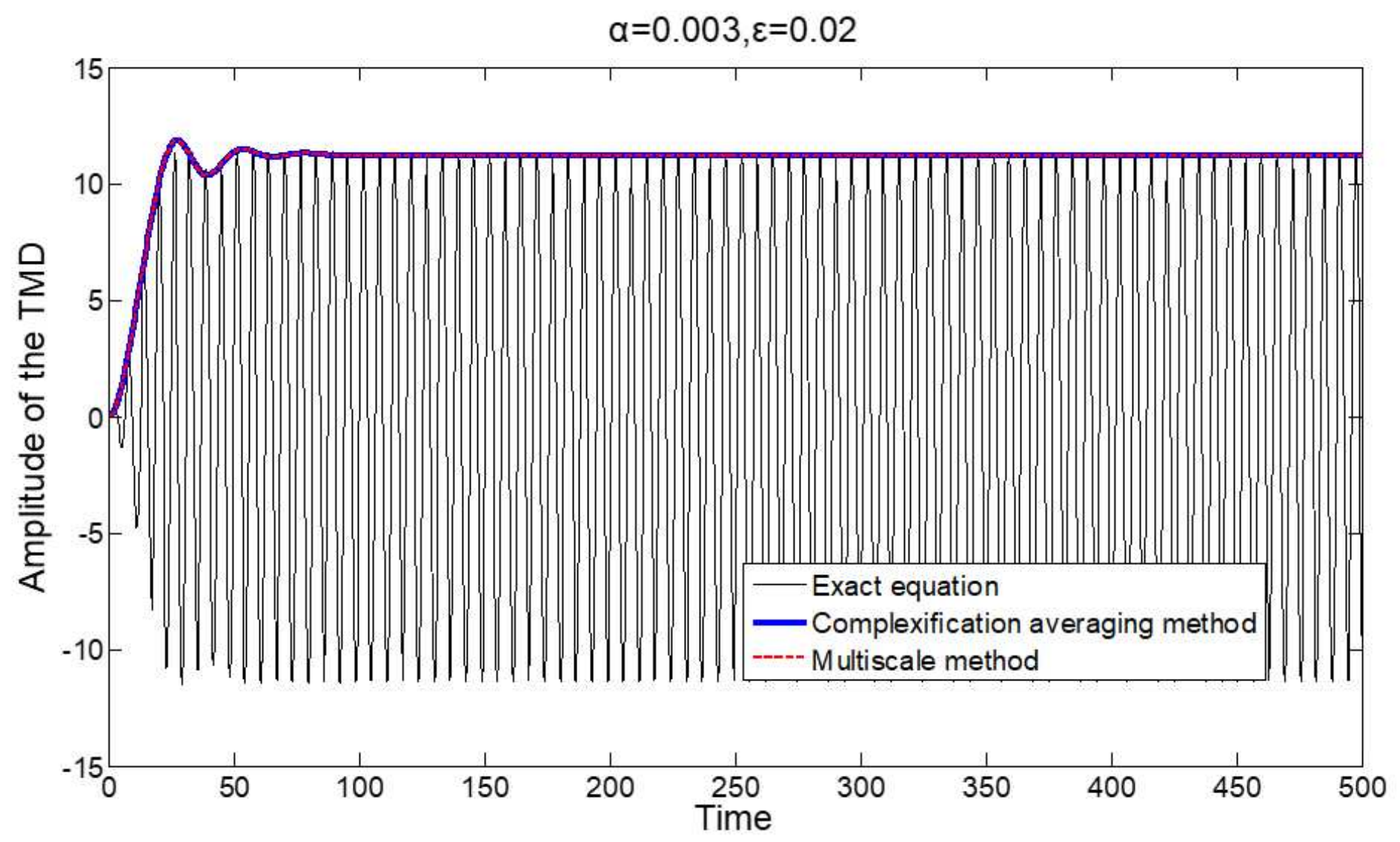

Figure 3

Time-history curves of the TMD for the case of $\varepsilon=0.02$ 


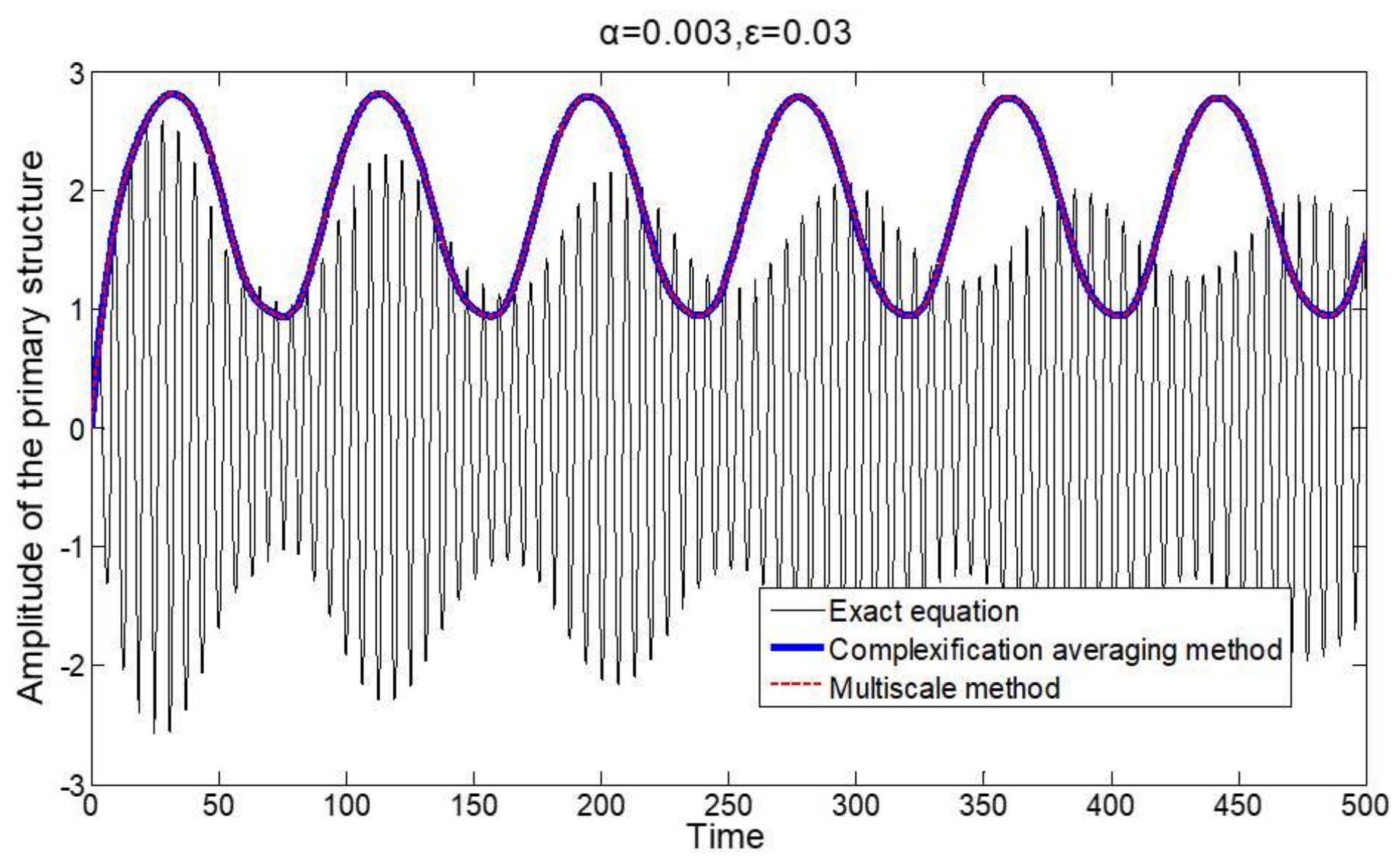

Figure 4

Time-history curves of the primary structure for the case of $\varepsilon=0.03$ 


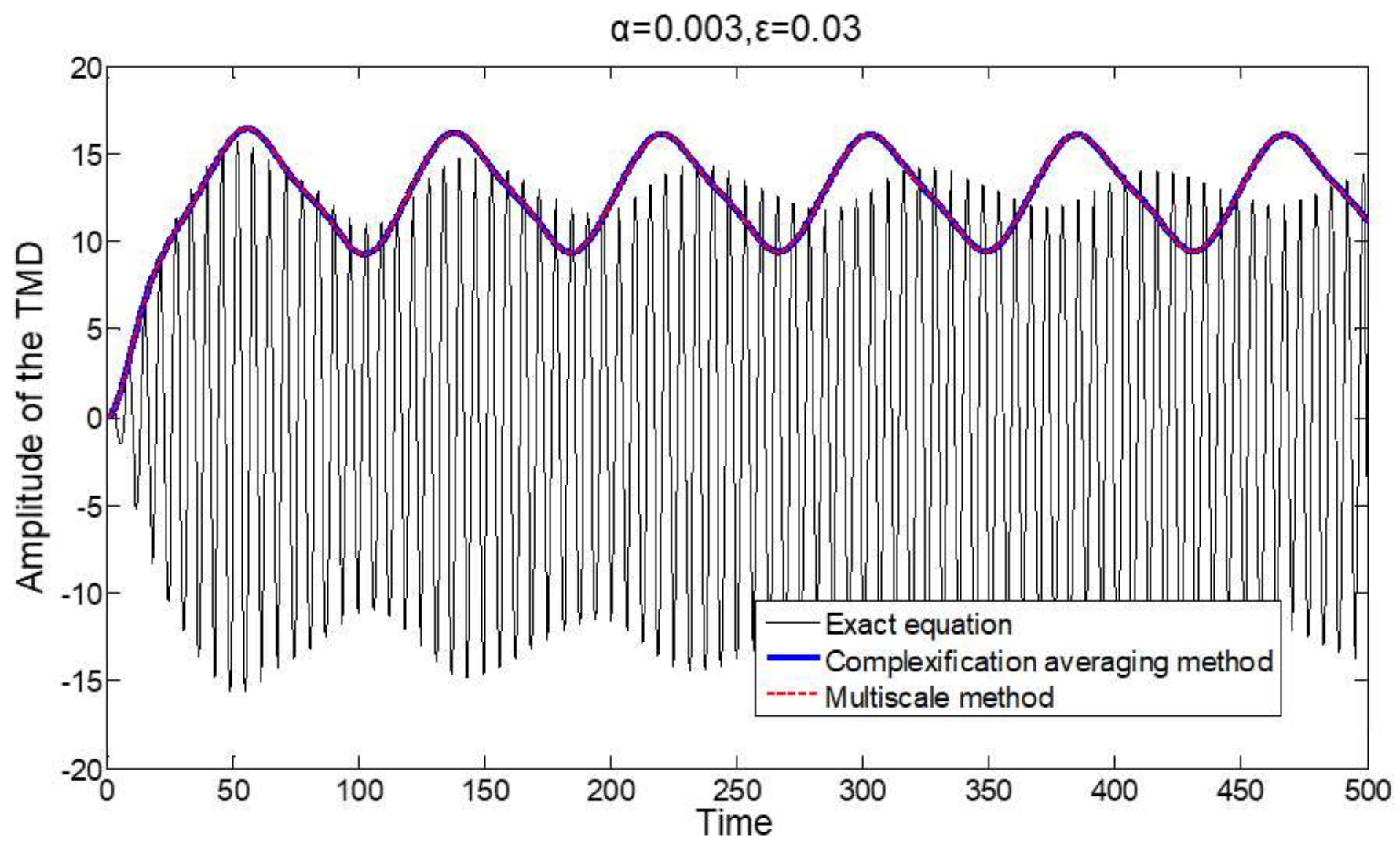

Figure 5

Time-history curves of the TMD for the case of $\varepsilon=0.03$ 


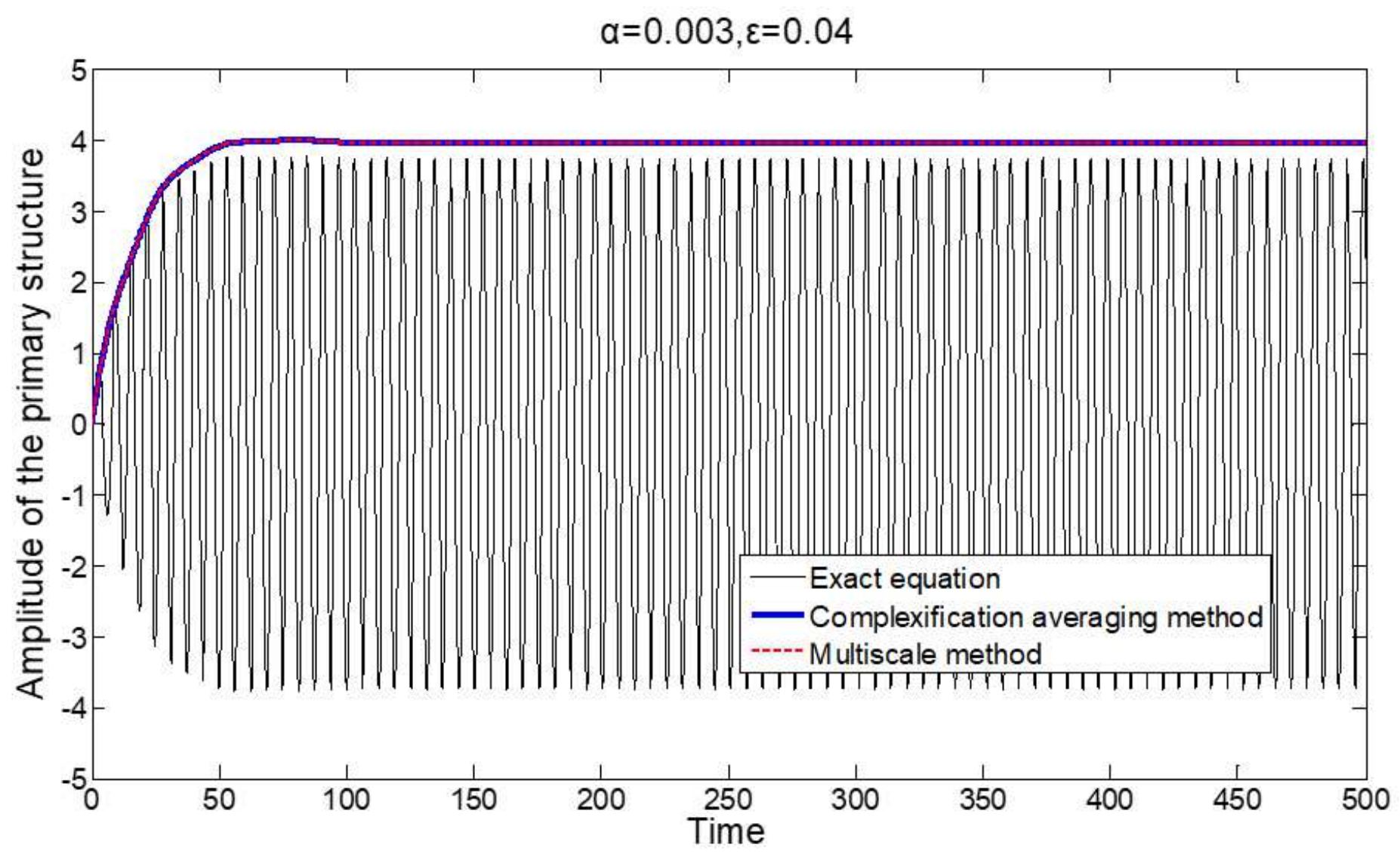

Figure 6

Time-history curves of the primary structure for the case of $\varepsilon=0.04$ 


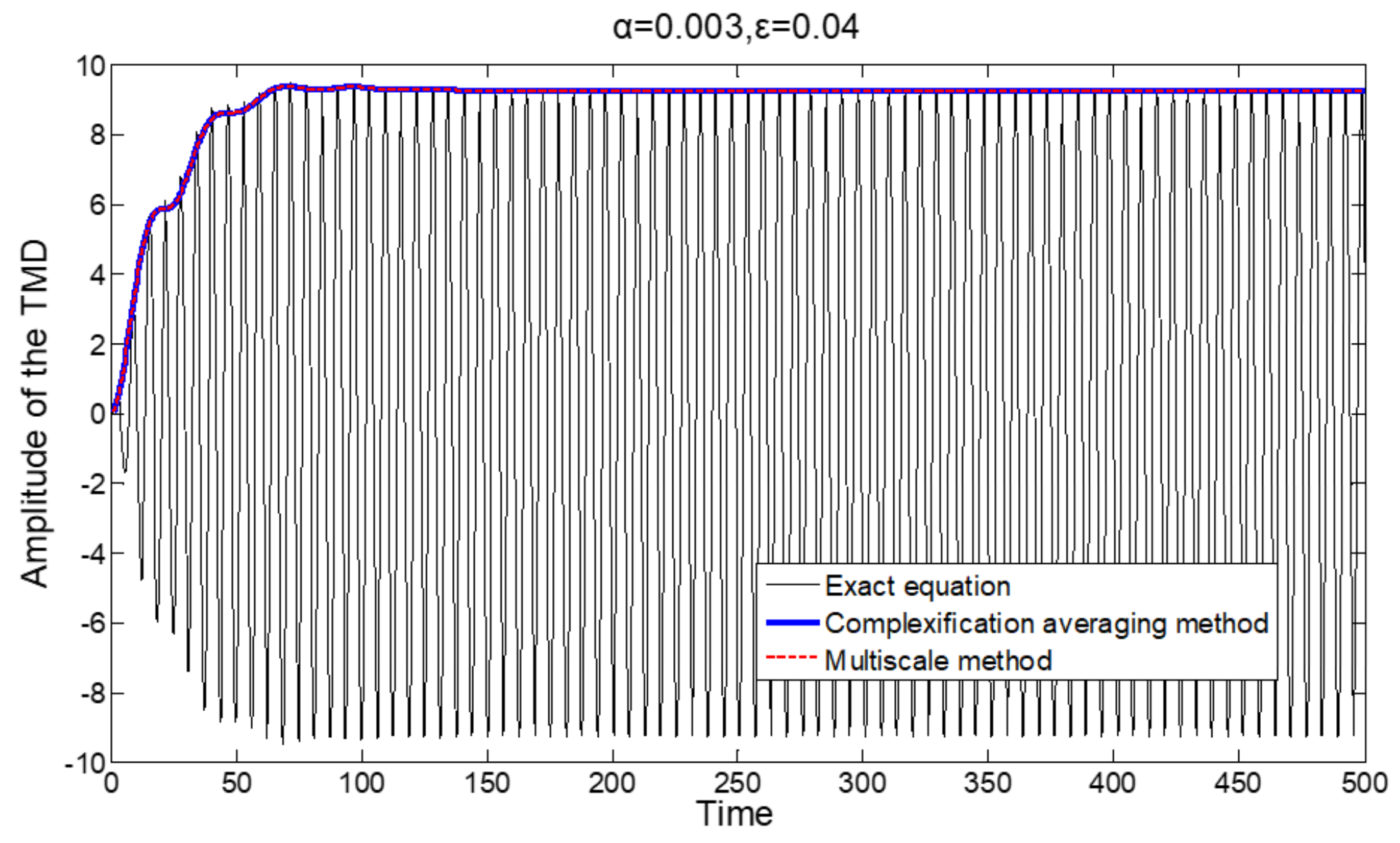

Figure 7

Time-history curves of the TMD for case $\varepsilon=0.04$ 


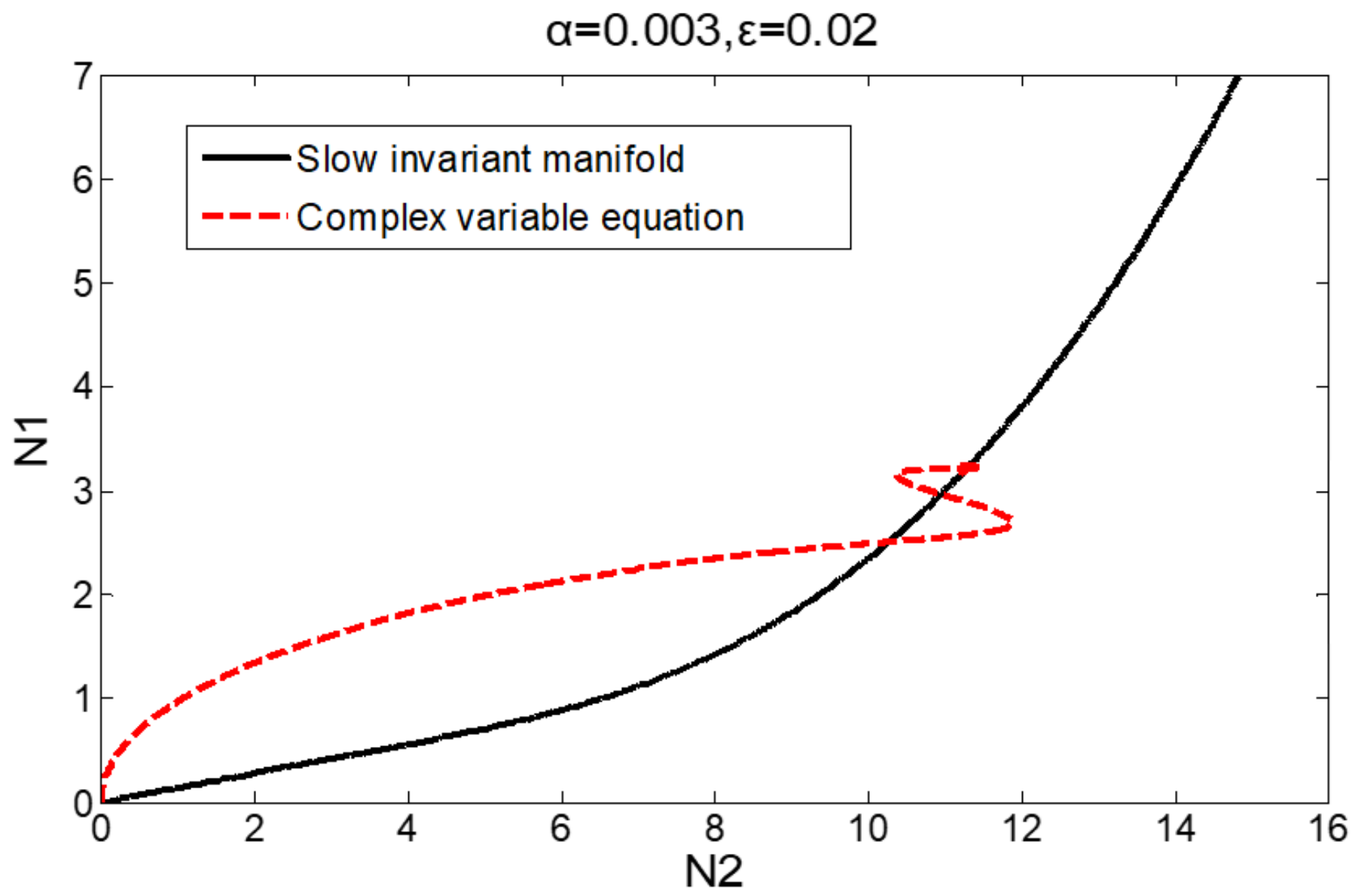

Figure 8

Slow invariant manifold for the case of $\varepsilon=0.02$ 


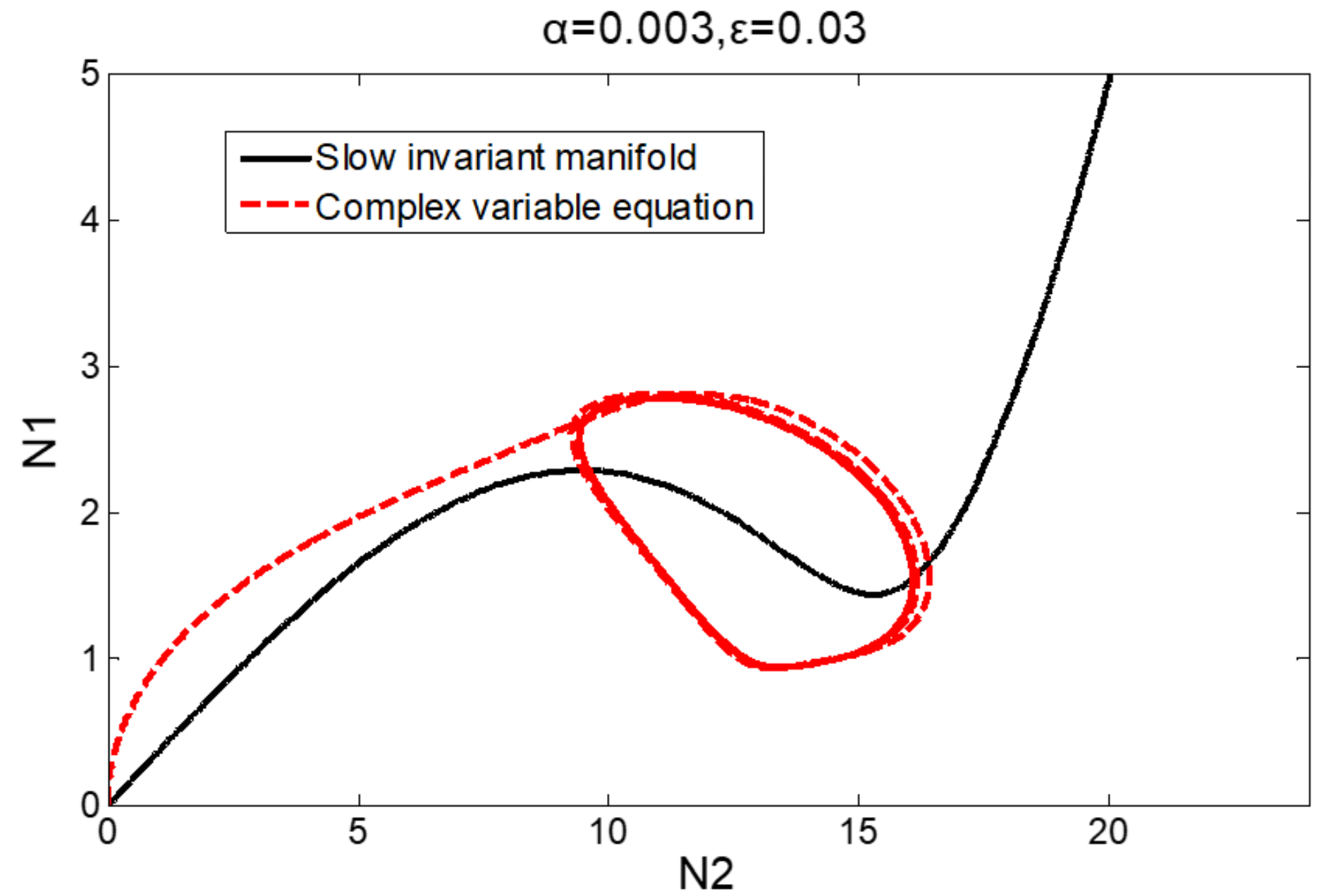

Figure 9

Slow invariant manifold for the case of $\varepsilon=0.03$ 


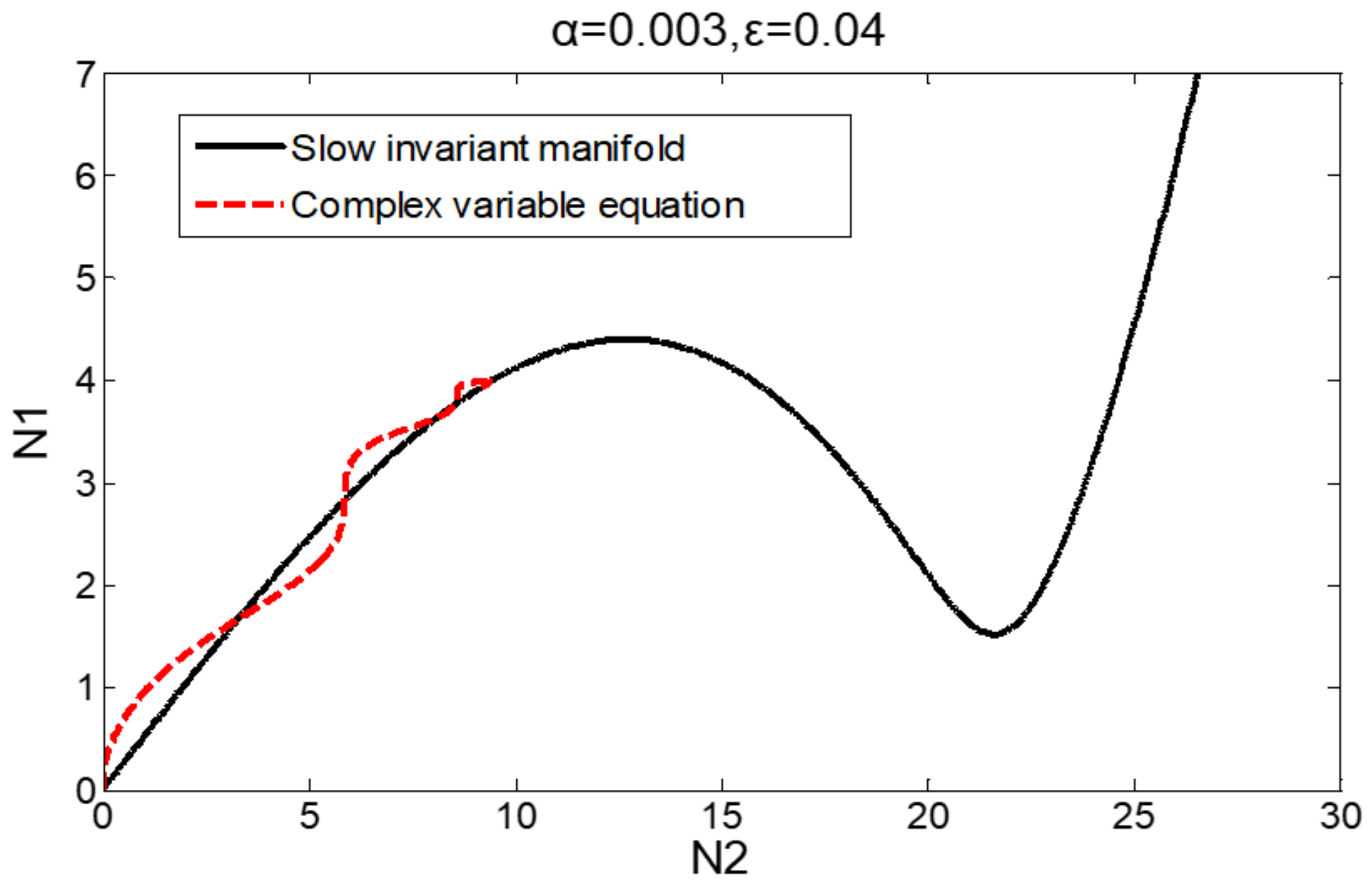

Figure 10

Slow invariant manifold for the case of $\varepsilon=0.04$ 


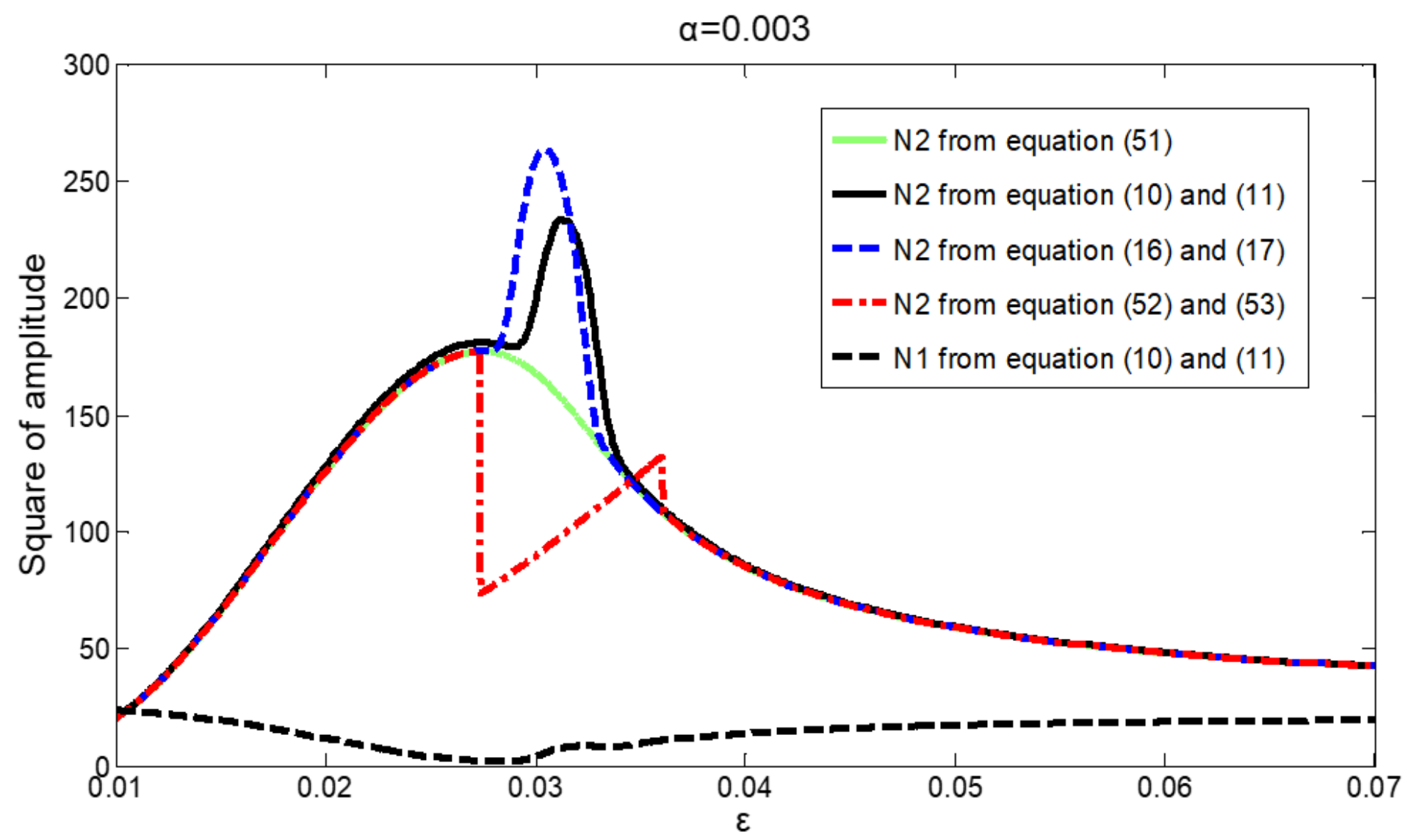

Figure 11

Relationship between mass ratio and amplitudes for the case of $a=0.003$

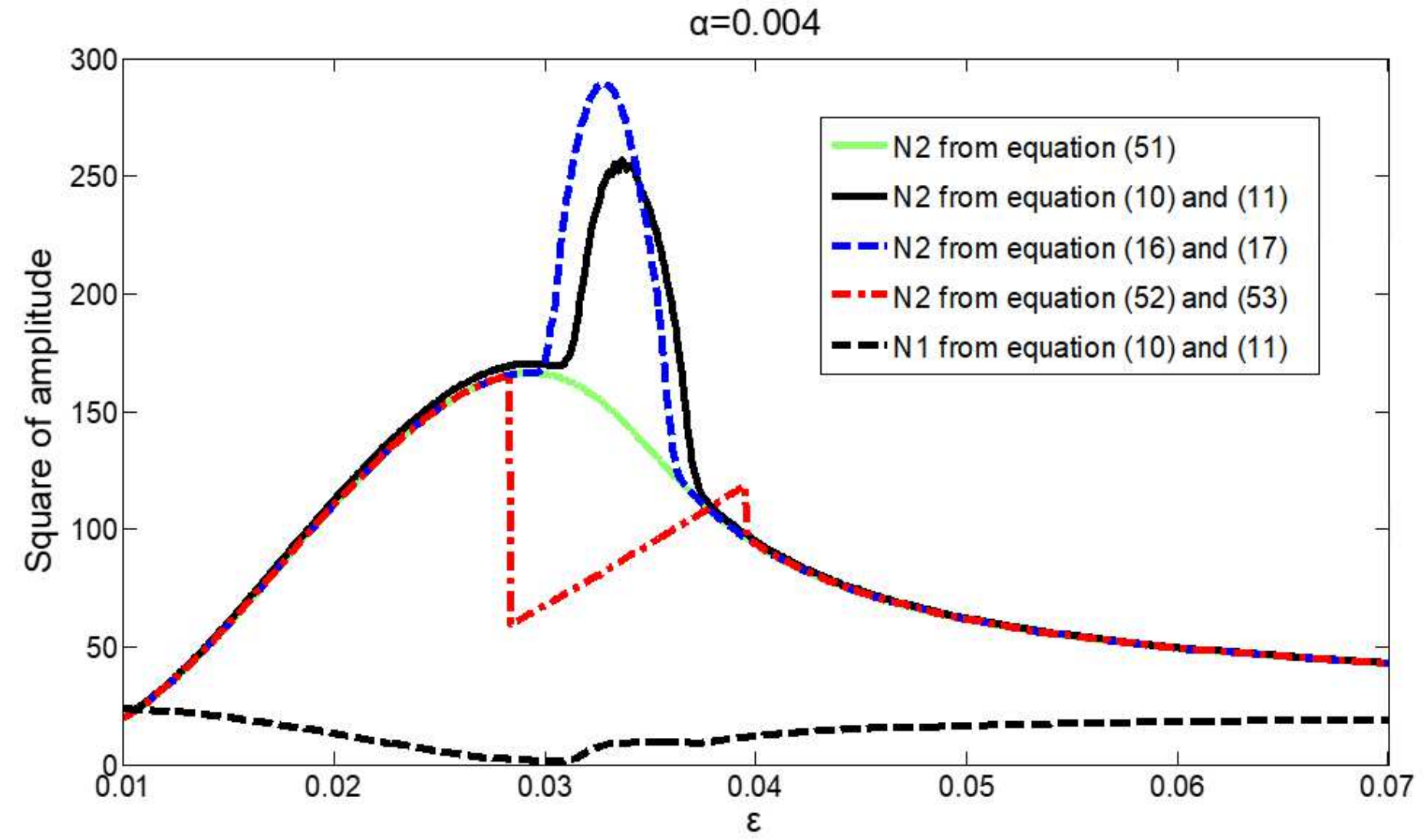


Figure 12

Relationship between mass ratio and amplitudes for the case of $a=0.004$

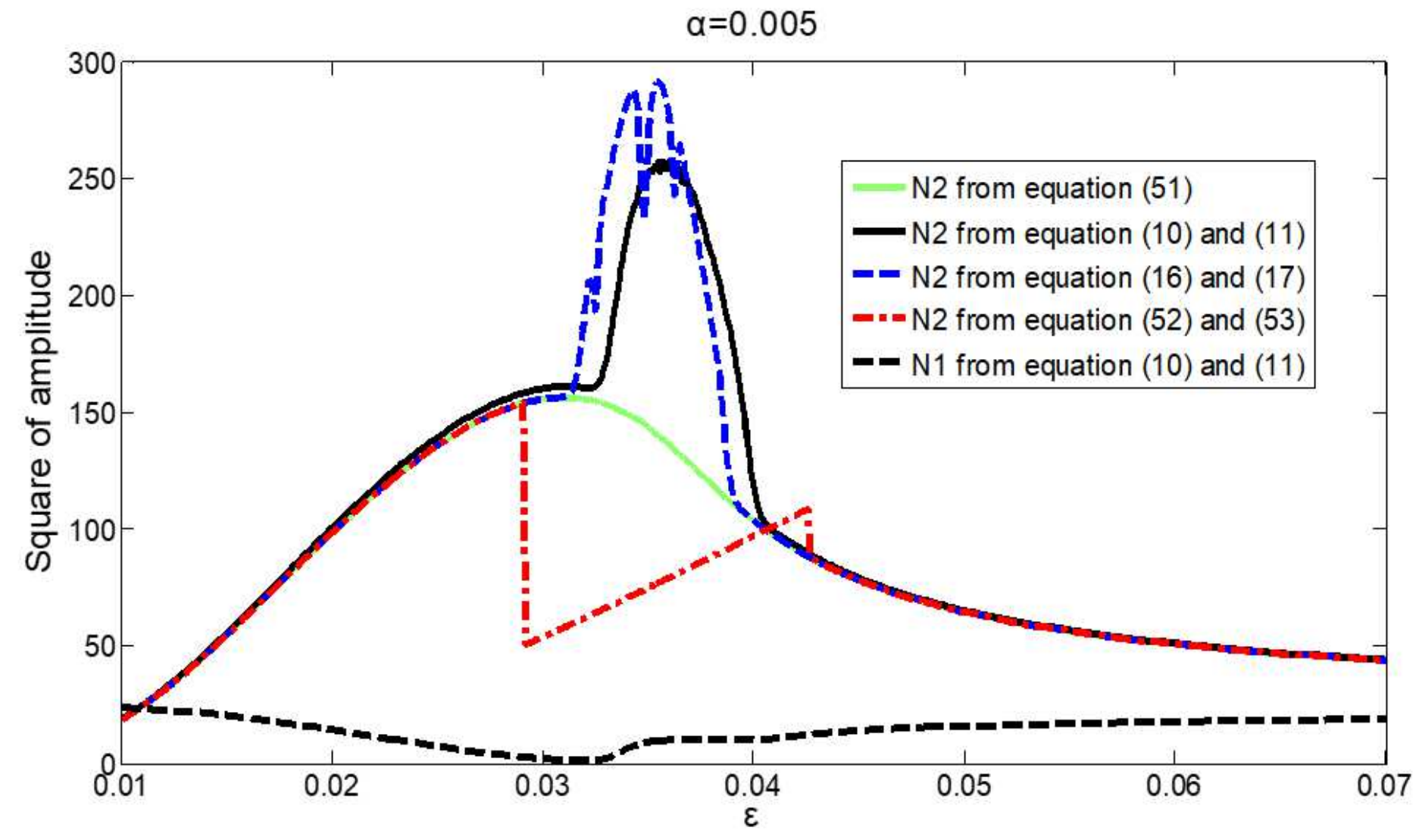

Figure 13

Relationship between mass ratio and amplitudes for the case of $a=0.005$ 


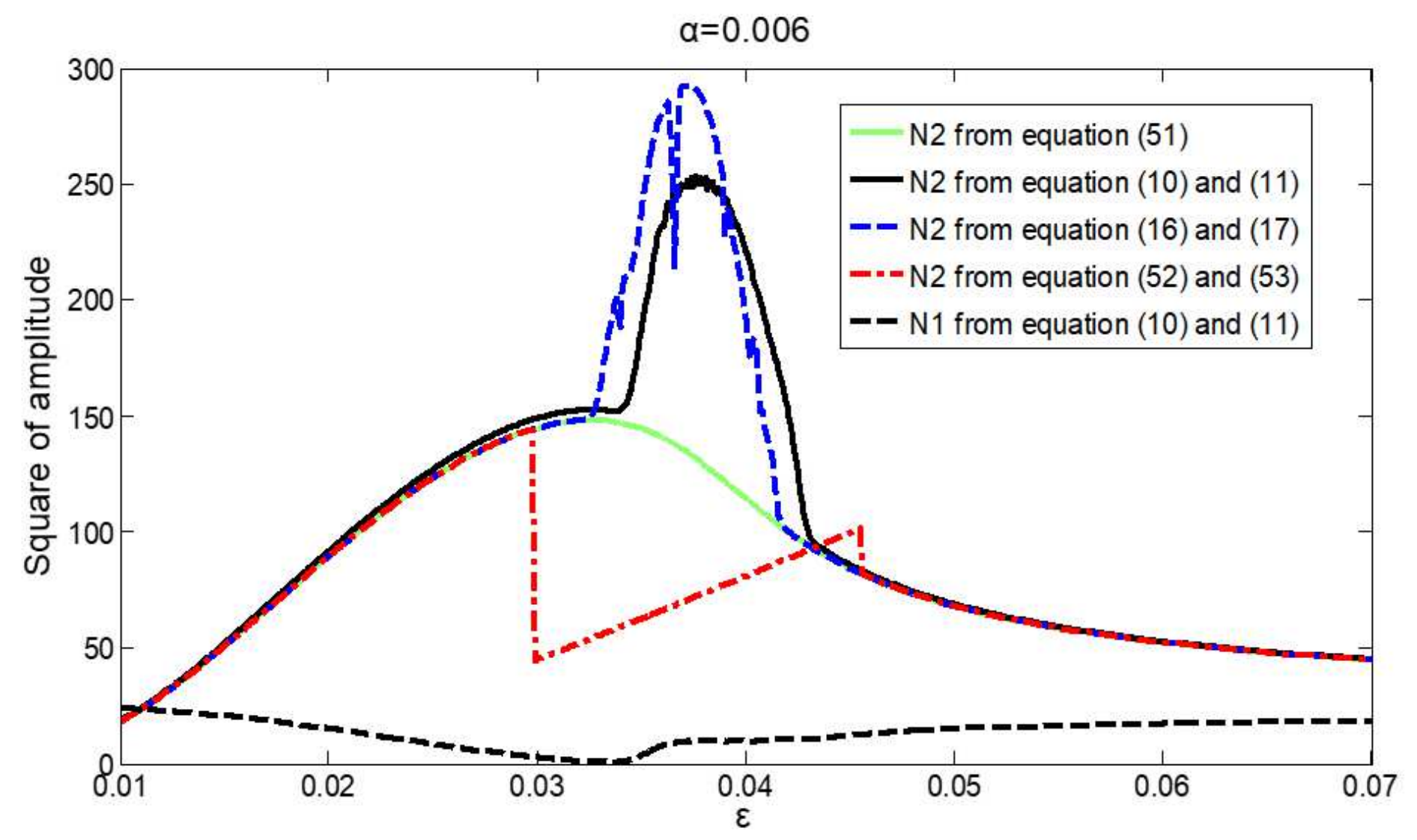

Figure 14

Relationship between mass ratio and amplitudes for the case of $a=0.006$ 


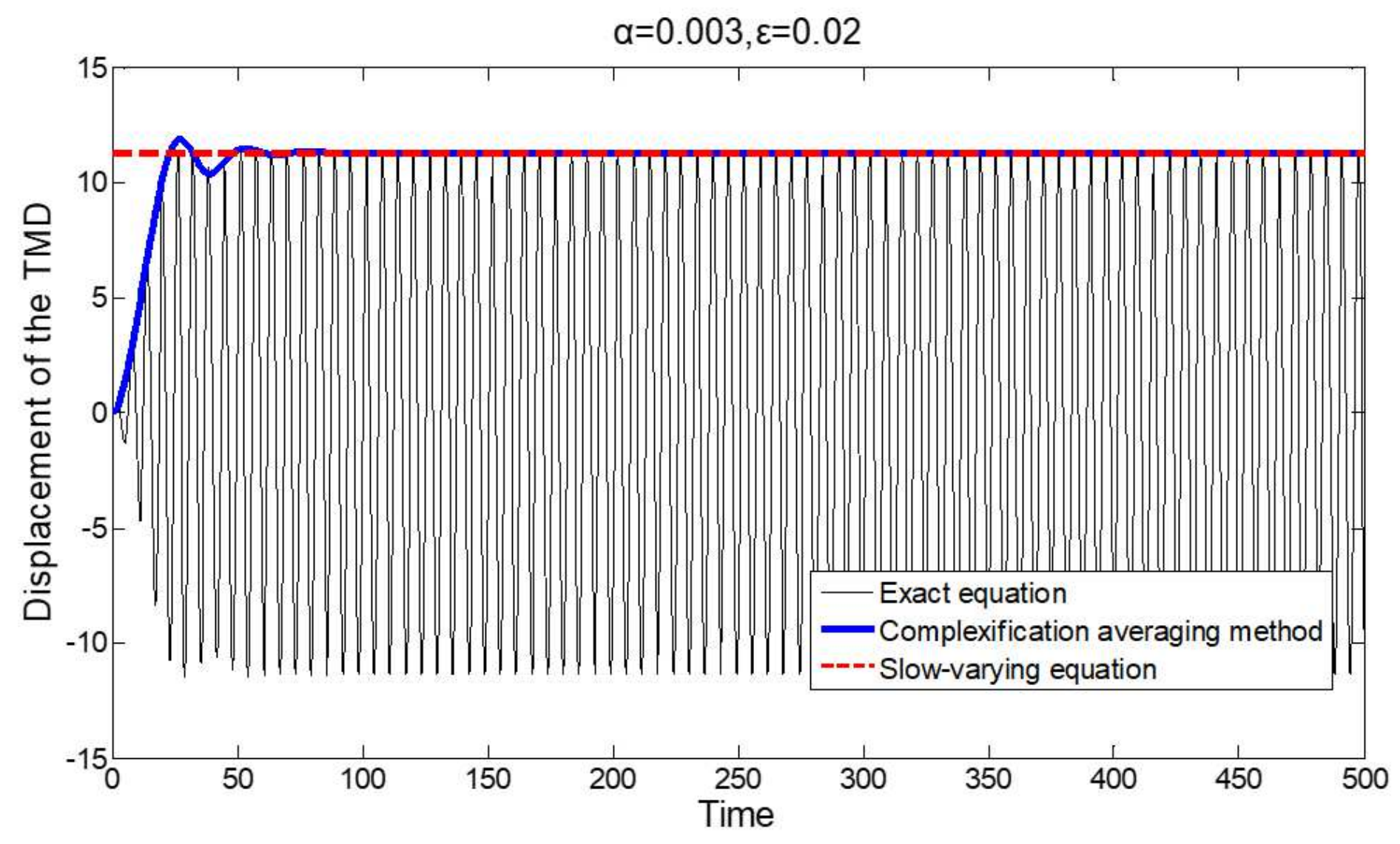

Figure 15

Time-history curves of the primary structure for the case of $\varepsilon=0.02$

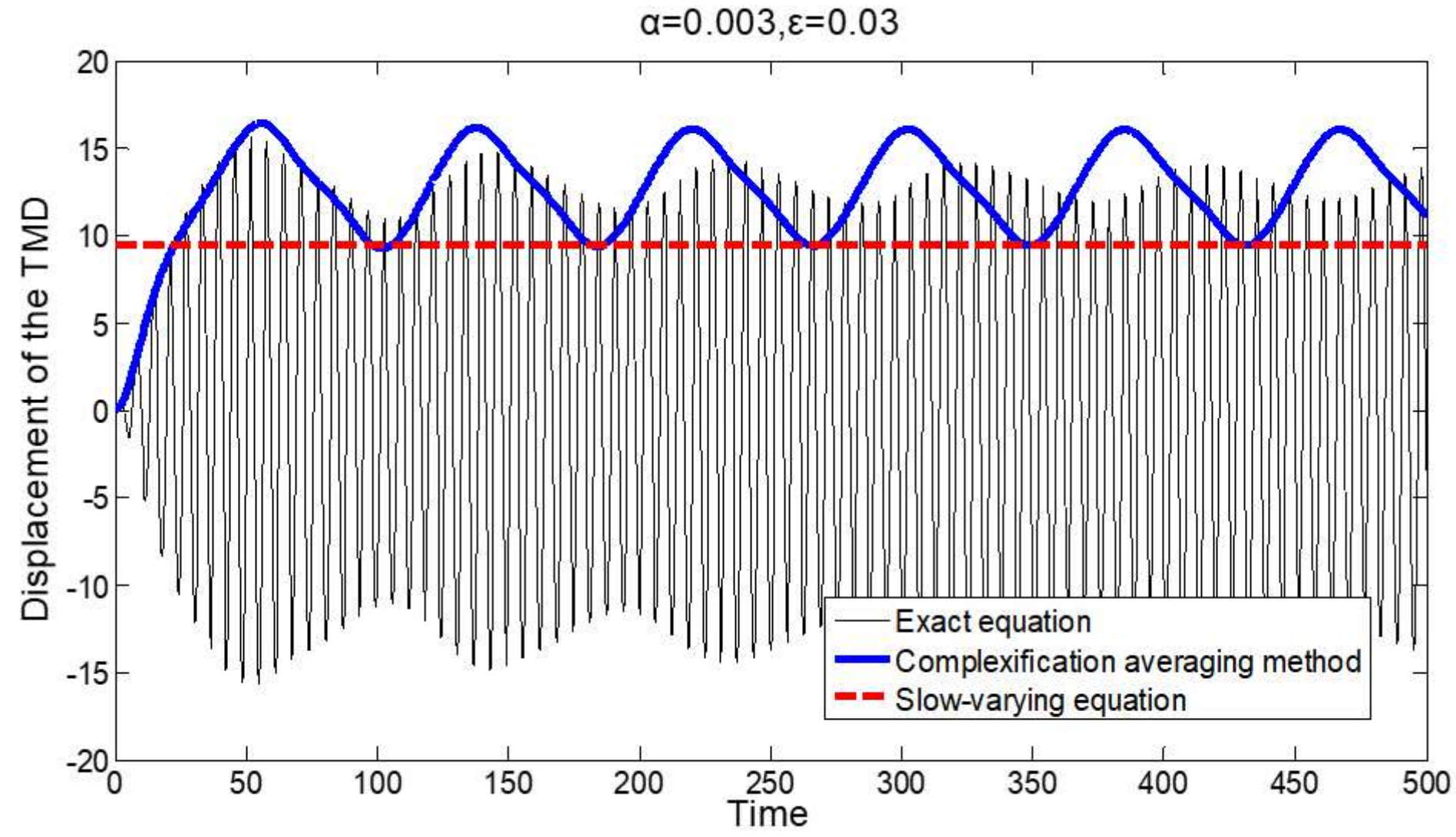


Figure 16

Time-history curves of the primary structure for the case of $\varepsilon=0.03$

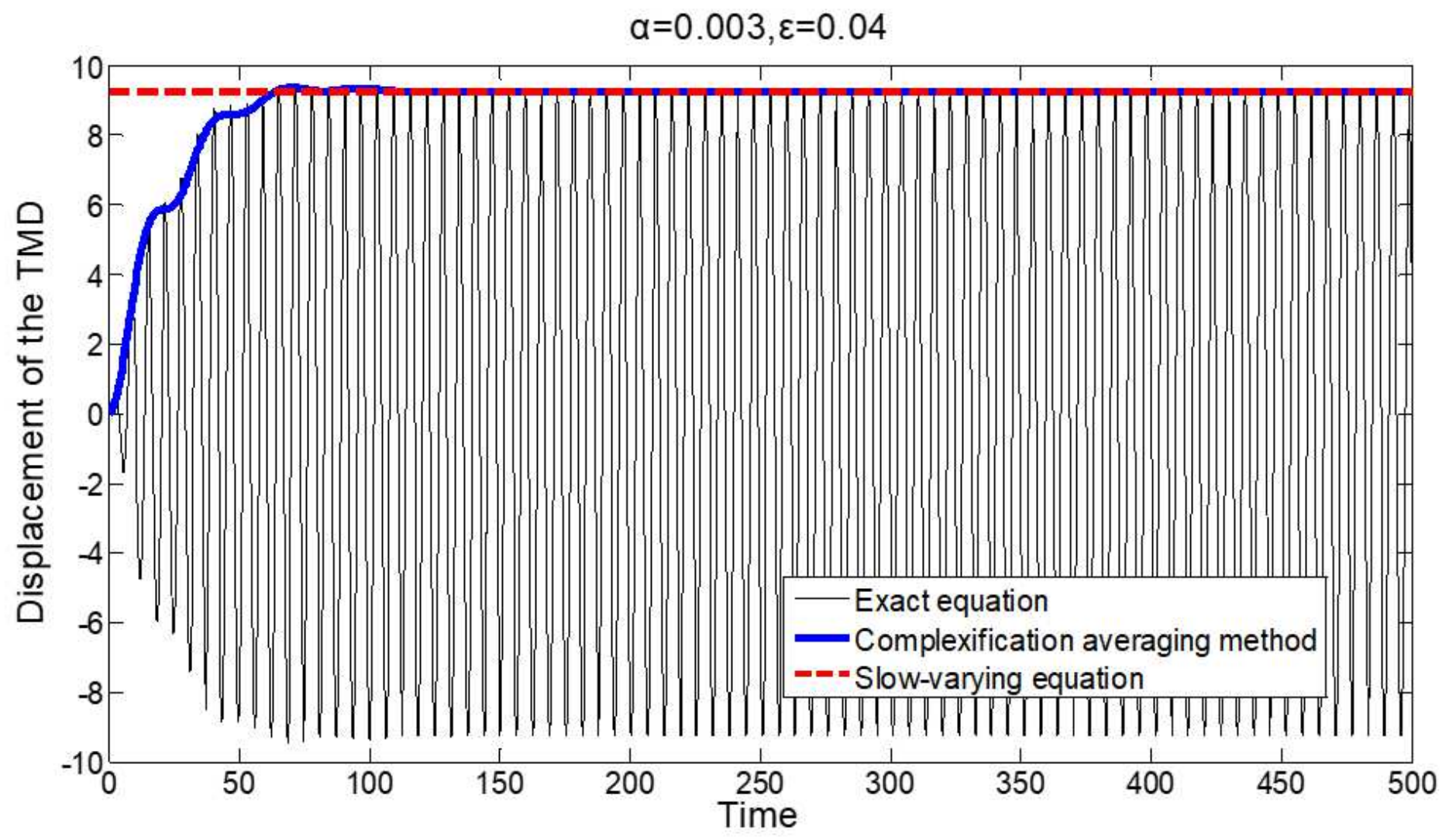

Figure 17

Time-history curves of the primary structure for the case of $\varepsilon=0.04$ 


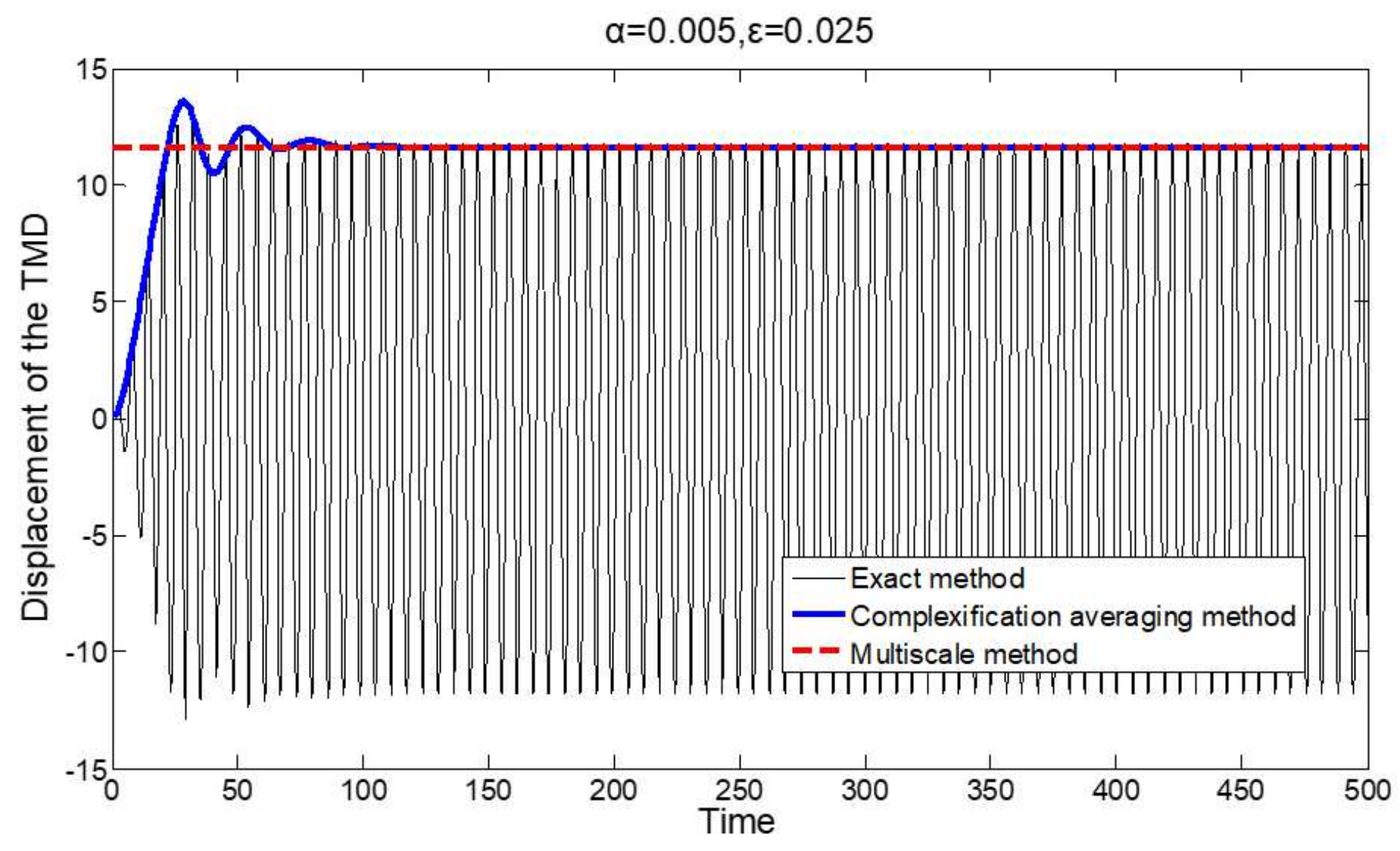

Figure 18

Time-history curves of the primary structure for the case of $a=0.005, \varepsilon=0.025$

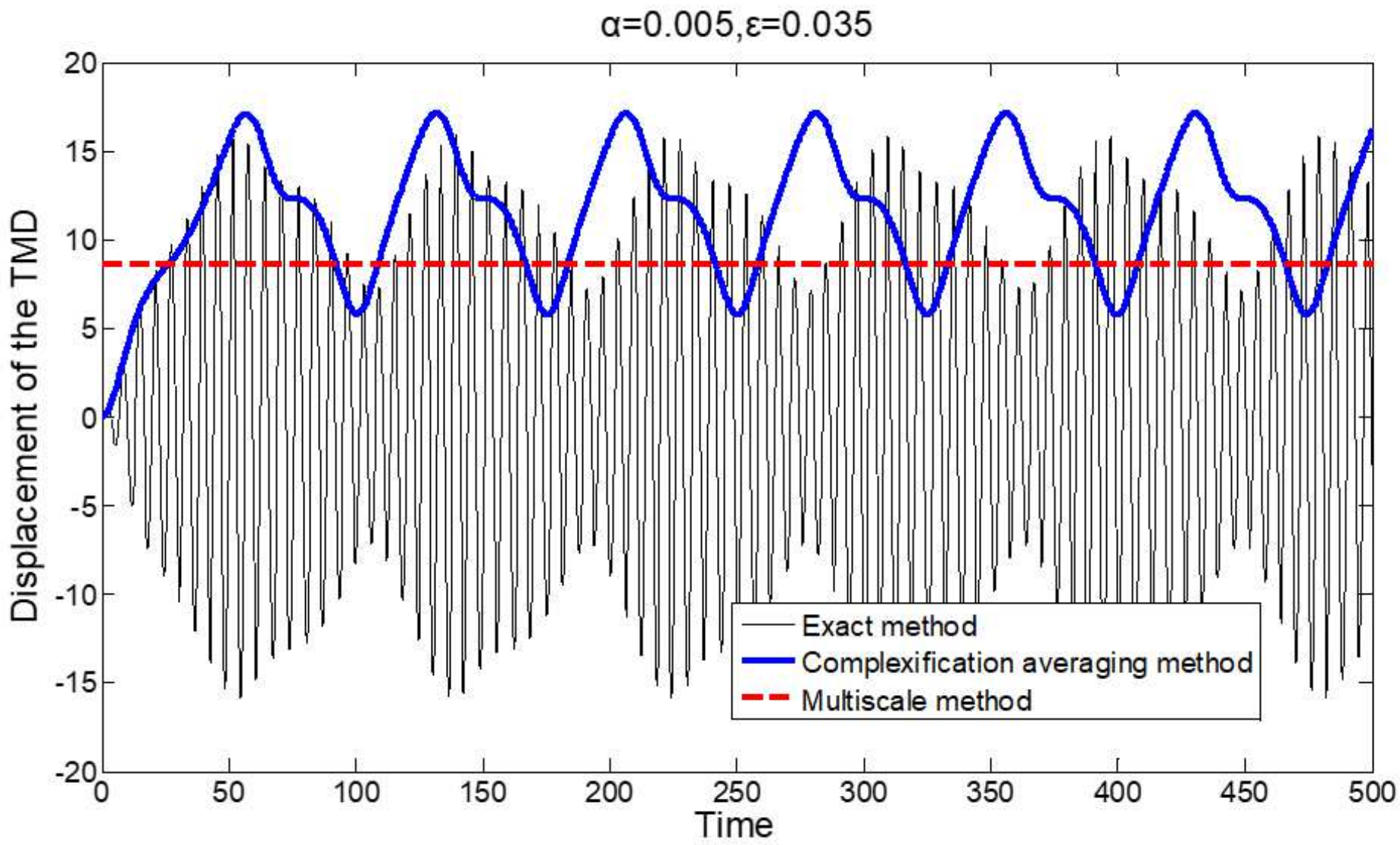


Figure 19

Time-history curves of the primary structure for the case of $a=0.005, \varepsilon=0.035$

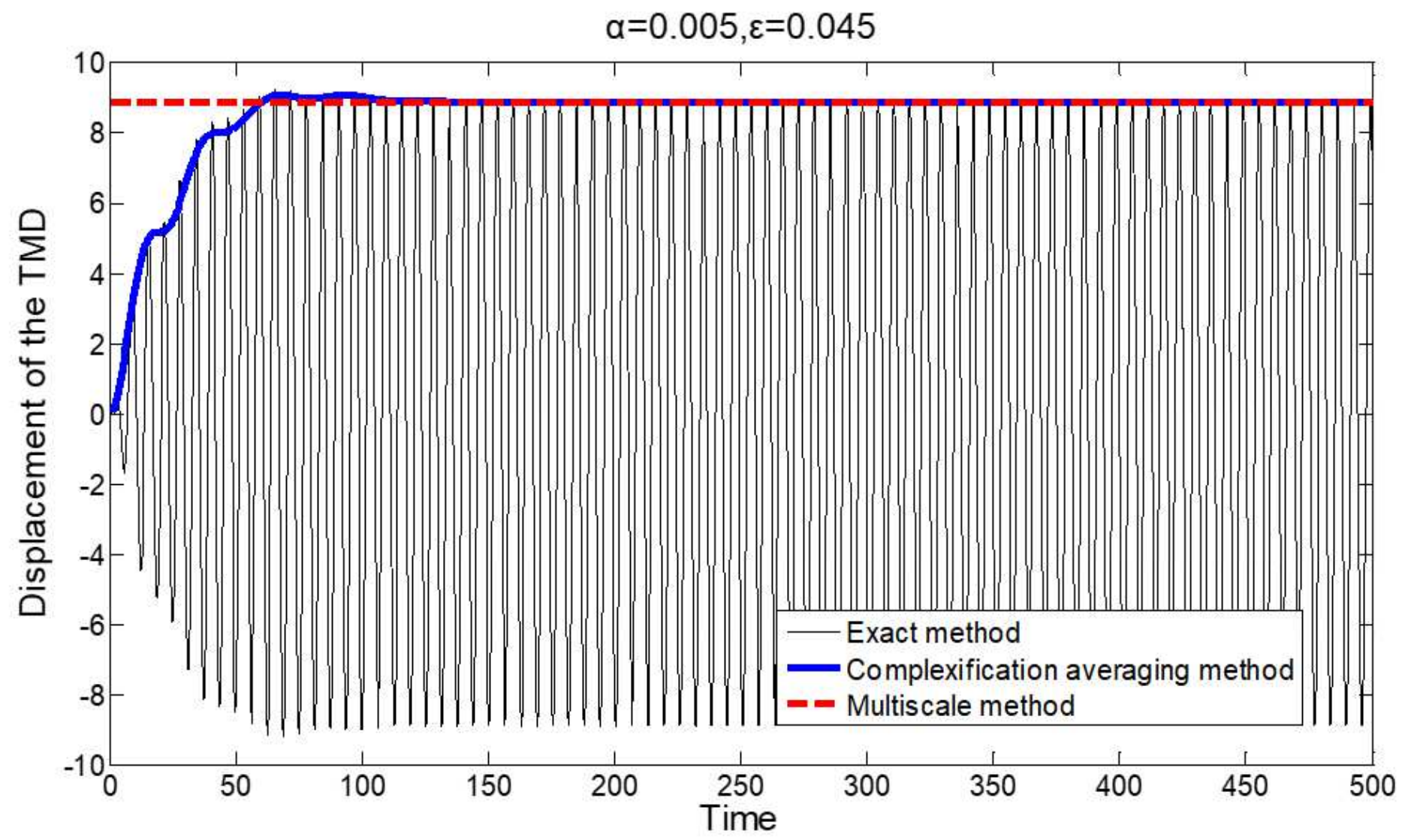

Figure 20

Time-history curves of the primary structure for the case of $a=0.005, \varepsilon=0.045$ 


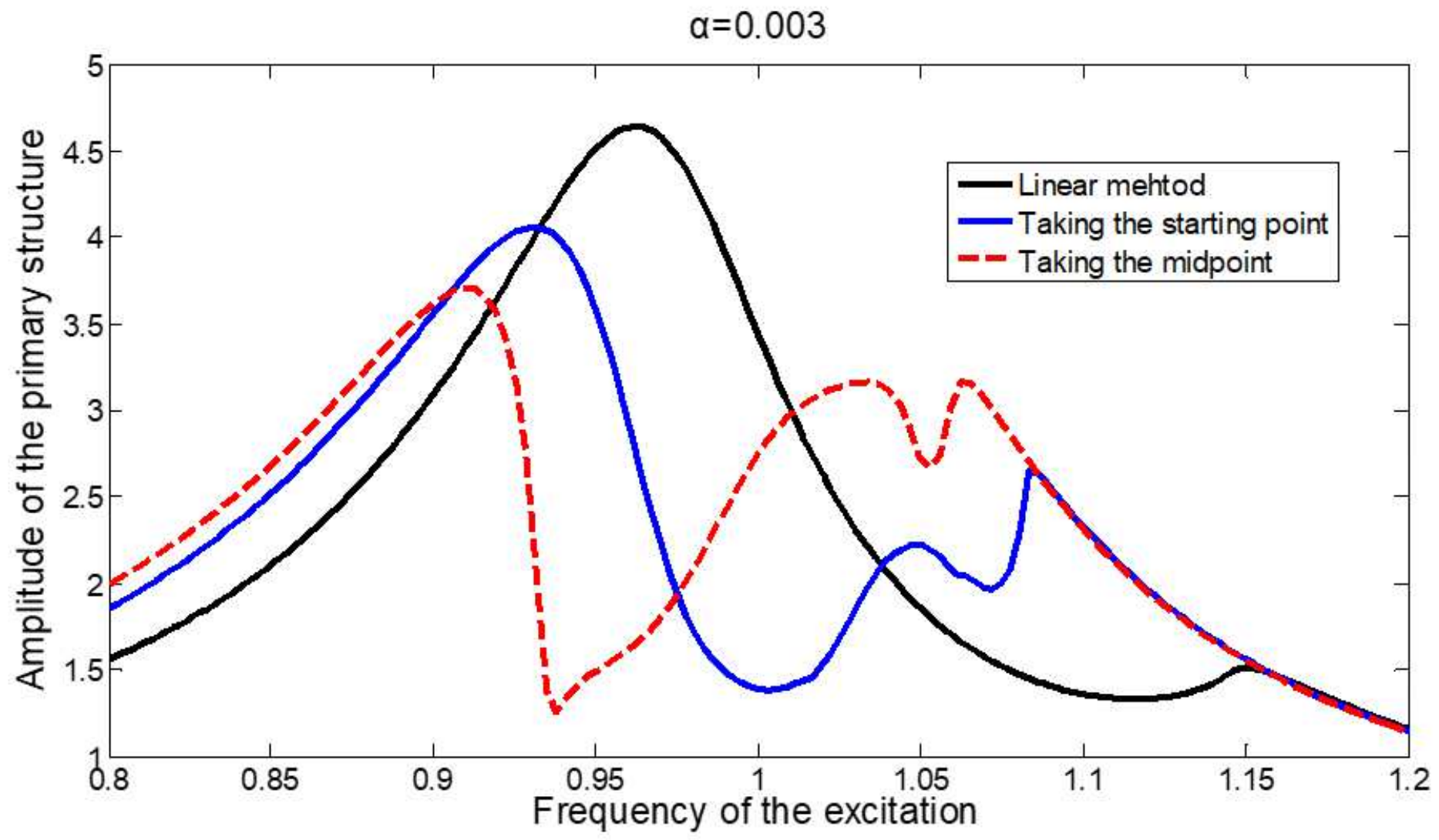

Figure 21

Frequency response curve of primary structure for the case of $a=0.003$

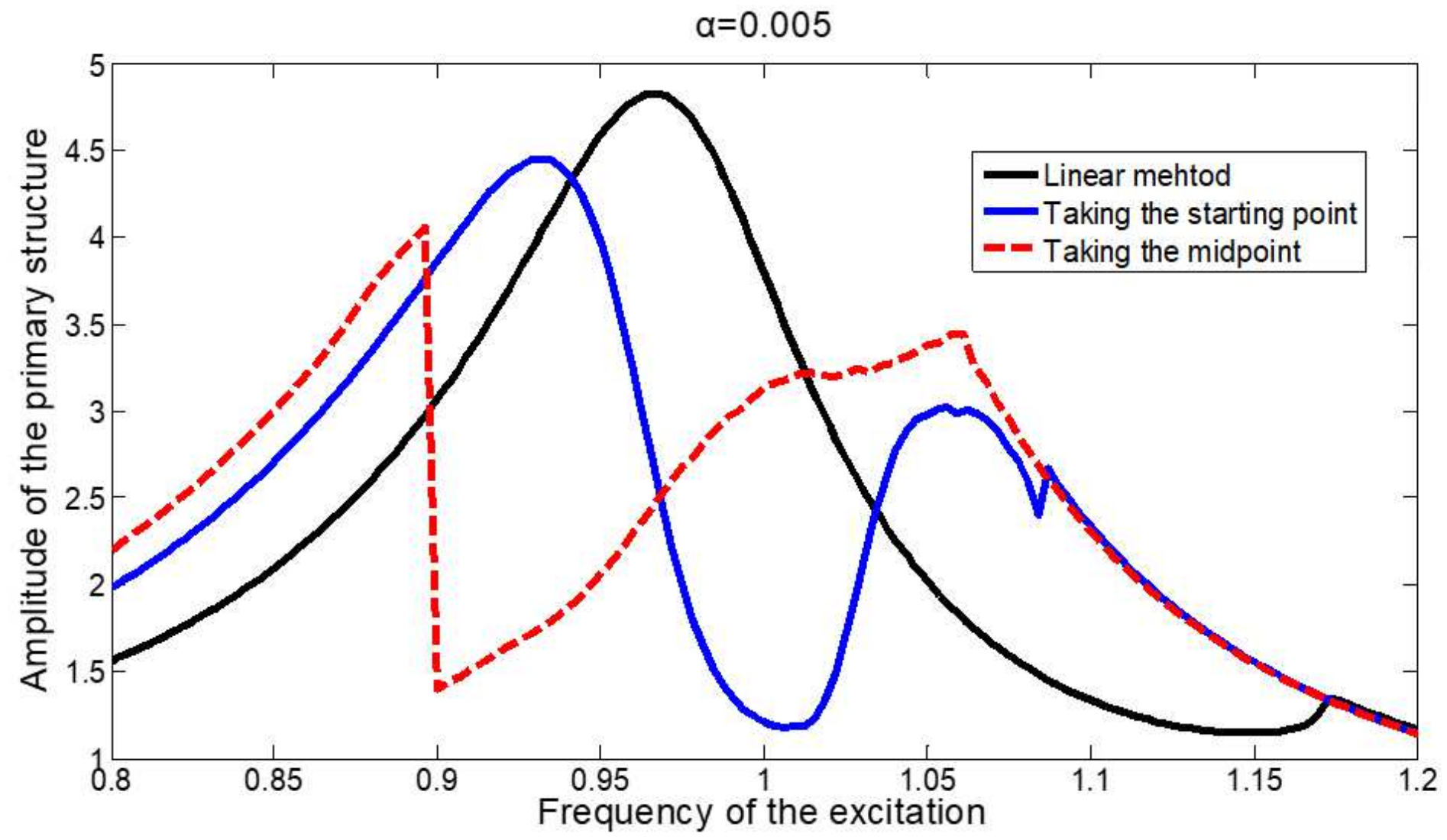


Figure 22

Frequency response curve of primary structure For the case of $a=0.005$

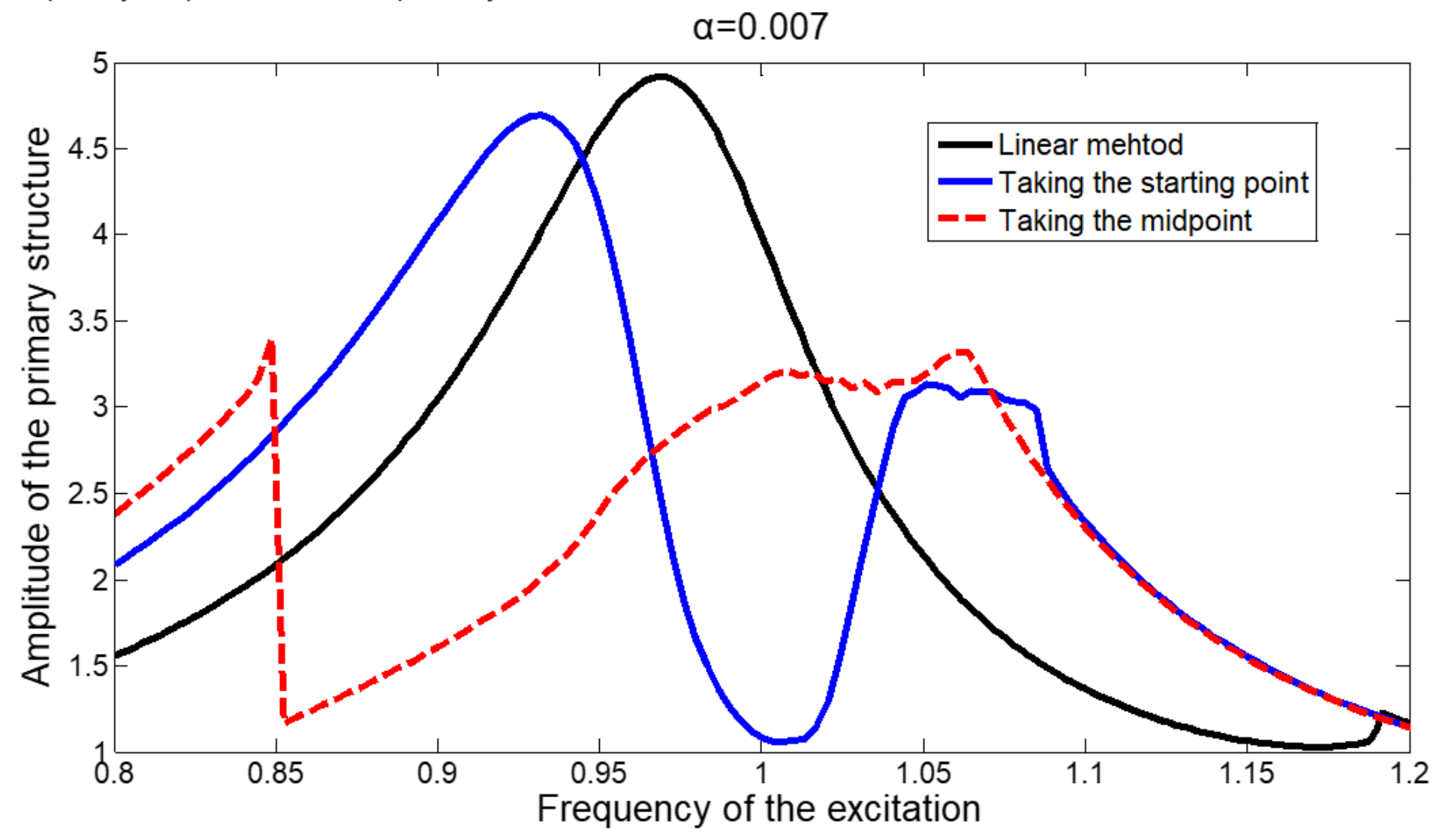

Figure 23

Frequency response curve of primary structure for the case of $a=0.007$ 